Research Article

\title{
Modified Theories of Gravitation behind the Spacetime Deformation
}

\author{
Gagik Ter-Kazarian \\ Ambartsumian Byurakan Astrophysical Observatory, Aragatsotn, 378433 Byurakan, Armenia \\ Correspondence should be addressed to Gagik Ter-Kazarian; gago_50@yahoo.com
}

Received 6 June 2014; Revised 1 September 2014; Accepted 11 September 2014

Academic Editor: Bennie F. L. Ward

Copyright (C) 2015 Gagik Ter-Kazarian. This is an open access article distributed under the Creative Commons Attribution License, which permits unrestricted use, distribution, and reproduction in any medium, provided the original work is properly cited.

\begin{abstract}
In the framework of proposed theory of spacetime deformation/distortion, we have a way to deform the spacetime through a nontrivial choice of the distortion-complex, displaying different connections, which may reveal different post-Riemannian spacetime structures as corollary. We extend this theory to address, in particular, the gauge model of the most general metricaffine gravity carrying both nontrivial torsion and nonmetricity. This model is constructed in the framework of the first order Lagrangian expressed in terms of the gauge potentials and their first derivatives. The equations of the standard theory, which have no propagating modes for torsion, can be equivalently replaced in modified framework by the modified equations, which in the limit of reducing the affine group leads to the modified Einstein-Cartan theory with dynamical torsion and beyond. In testing the modified framework for various particular cases, we use the Lagrange multipliers for extinguishing nonmetricity and torsion.
\end{abstract}

\section{Introduction}

We will not attempt a history of gauge theory of gravitation in general, but only of those that seem most relevant to the particular theory of this paper. From its historical development, the efforts in gauge treatment of gravity mainly focus on the quantum gravity and microphysics, with the recent interest, for example, in the theory of the quantum superstring or, in the very early universe, in the inflationary model. The papers on the gauge treatment of gravity provide a unified picture of gravity modified models based on several Lie groups [1-61]. However, currently no single theory has been uniquely accepted as the convincing gauge theory of gravitation which could lead to a consistent quantum theory of gravity. They have evoked the possibility that the treatment of spacetime might involve non-Riemannian features on the scale of the Planck length. This necessitates the study of dynamical theories involving post-Riemannian geometries. On the other hand, a general way to deform the spacetime metric with constant curvature has been explicitly posed by [62-66]. The problem was initially solved only for $3 \mathrm{D}$ spaces, but consequently it was solved also for spacetimes of any dimension. It was proved that any semi-Riemannian metric can be obtained as a deformation of constant curvature matric, with this deformation being parameterized by a 2 form. A novel definition of spacetime metric deformations, parameterized in terms of scalar field matrices, is proposed by [67]. However, without care of the historical justice and authenticity, it also should be emphasized that the continuous deformations within the space of connections were studied by $[17,18]$. This concept of a deformation seems to be a special case of a prolongation. A main idea is as follows: since any tensor-valued one-form transforms homogenously with respect to linear gauge transformations, its subtraction from a connection can be regarded as a continuous deformation within the space of connections. A more general approach is suggested by [68], whereas the authors present the extension to $4 \mathrm{D}$ of an euclidean $2 \mathrm{D}$ model that exhibits spontaneous generation of a metric. In this model gravitons emerge as Goldstone bosons of a global $S O(D) \times G L(D)$ symmetry broken down to $S O(D)$. They formulated microscopic theory without having to appeal to any particular spacetime metric and only assume the preexistence of a manifold endowed with an affine connection. Moreover, here it seems that not even a flat metric needs to be assumed. In this sense the microscopic theory is quasitopological. The vierbein appears as a condensate of the fundamental fermions. In spite of having nonstandard characteristics, the microscopic theory 
appears to be renormalizable. Recently, some clarifications are also collected in the book [69], where the authors have tried to collect the established results and to focus new investigations on the real loopholes of the theory. Only time will tell whether any of these intriguing theories is correct and which of the hypothesized hidden symmetries is actually realized in nature.

It is well known that the notions of space and connections should be separated; see, for example, $[13,70-72]$. The curvature and torsion are in fact properties of a connection, and many different connections are allowed to exist in the same spacetime. Therefore, when considering several connections with different curvature and torsion, one takes spacetime simply as a manifold and connections as additional structures. Allowing minimal departure from semi-Riemannian geometry would consist in admitting torsion. The concept of a linear connection as an independent and primary structure of spacetime is the fundamental proposal put forward by Cartan's geometrical analysis [43-46], whereas he gave a beautiful geometrical interpretation of torsion representing a translational misfit. The Einstein-Cartan (EC) theory also called Einstein-Cartan-Sciama-Kibble theory is the minimal extension of the general relativity, which considers curvature and torsion as representing independent degrees of freedom. In the standard EC theory, the equation defining torsion is of algebraic type, and not a differential equation, and no propagation of torsion is allowed. Even at the textbook level it was common knowledge known from the weak interaction that the causality reasons do not respect a contact interaction. Therefore, many modifications of the EC theory have been proposed in recent years; see, for example, [37, 73-79], but all these approaches are subject to many uncertainties. In particular, in [80] the authors analyze the functional renormalization group flow of quantum gravity on the EC theory space. The latter consists of all action functionals depending on the spin connection and the vielbein field (coframe) which are invariant under both spacetime diffeomorphisms and local frame rotations. They find evidence for the existence of at least one non-Gaussian renormalization group fixed point which seems suitable for the asymptotic safety construction in a setting where the spin connection and the vielbein are the fundamental field variables. The difference between Einstein's general relativity and its Cartan extension is analyzed within the scenario of asymptotic safety of quantum gravity by [81], whereas it was found that the four-fermion interaction distinguishes the Einstein-Cartan theory from its Riemannian limit. However, modifications of EC theory are intricate. In a recent paper [82], we construct the two-step spacetime deformation (TSSD) theory which generalizes and, in particular cases, fully recovers the results of the conventional theory of spacetime deformation [62-66]. Conceptually and techniquewise the TSSD theory is versatile and powerful and manifests its practical and technical virtue in the fact that through a nontrivial choice of explicit form of a world-deformation tensor, which we have at our disposal, in general, we have a way to deform the spacetime displaying different connections, which may reveal different post-Riemannian spacetime structures as corollary: (1) the Weitzenböck spacetime structure- $\left(W_{4}\right)$ underlying a teleparallelism theory of gravity, see, for example, [23, $24,38,83-93]$; (2) the RC manifold- $\left(U_{4}\right)$ underlying EC theory, for a comprehensive references, see, for example, [34$37,51,52,94]$; (3) or even the most general linear connection of metric-affine gravity (MAG) taking values in the Lie algebra of the $4 \mathrm{D}$-affine group, $A(4, R)=R^{4} \otimes G L(4, R)$; see, for example, [15-18, 42-50, 55-57]. This represents the semidirect product of the group of $4 \mathrm{D}$-translations and general linear 4D-transformations. Continuing along this line, in the present paper, we address the essential features of the gauge model of the most general MAG theory in context of TSSD-construction of post-Riemannian geometry, whereas to complete the TSSD theory, we build up the distortion-complex (DC) and show how it restores the worlddeformation tensor, which still has been put in [82] by hand. A formulation of the major physical aspects of the modified gauge MAG theory will be given in the framework of the first order Lagrangian expressed in terms of the gauge potentials and their first derivatives. All the fundamental gravitational structures in fact, the metric as much as the coframes and connections, acquire a DC induced theoretical interpretation. There is another line of reasoning which supports the side of this model. We address the key problem of a dynamical torsion and show that the equations of the standard MAG theory can be equivalently replaced by the set of modified $M A G$ equations in which the torsion, in general, is turned out to be dynamical. We define the physical conditions for the spacetime deformations when the spin-spin interaction becomes short-range propagating. As an application we have to test the general TSSD-MAG framework in some limit, namely, we have to put on Lagrange multipliers to recover the TSSD versions of different (sub)cases of Poincaré gauge theory (PG), Einstein-Cartan theory, teleparallel gravity $\left(\mathrm{GR}_{\|}\right)$, and general relativity (GR). This allows amplifying and substantiating the assertions made in [82]. Moreover, imposing different physical constraints upon the spacetime deformations, in this modified framework we may reproduce the term in the well known Lagrangian of pseudoscalarphoton interaction theory, or terms in the Lagrangians of pseudoscalar theories [95-100]. This paper is organized as follows. To start with, in Section 2 we complete the spacetime deformation theory [82] by introducing DC complex and showing how it restores the world-deformation tensor. We also briefly revisit the theory of TSSD to make the rest of the paper understandable. An outline of the key points of TSSD theory of relevance to gauge MAG theory is stated in Section 3. The concluding remarks are presented in Section 4. The appendices discuss some relevant topics on the TSSD theory and algebraic operations in use.

\section{Spacetime Deformations and Beyond: Model Building}

Let us consider a smooth deformation map $\bar{\Omega}: M_{4} \rightarrow \mathscr{M}_{4}$, written in terms of the world-deformation tensor $\bar{\Omega}$, general, $\mathscr{M}_{4}$, and flat, $M_{4}$, smooth differential $4 \mathrm{D}$-manifolds. The tensor, $\bar{\Omega}$, can be written in the form $\bar{\Omega}:=\bar{D} \bar{f}\left(\bar{\Omega}_{l}^{m}:=\bar{D}_{\mu}^{m} \bar{f}_{l}^{\mu}\right)$, where the distortion-complex (DC) includes the invertible 
distortion matrix $\bar{D}\left(\bar{D}_{\mu}^{m}\right)$ and the tensor $\bar{f}\left(\bar{f}_{l}^{\mu}:=\partial_{l} x^{\mu}\right.$ and $\left.\partial_{l}:=\partial / \partial x^{l}\right)$. Here we use Greek alphabet $(\mu, \nu, \rho, \ldots=$ $0,1,2,3)$ to denote the holonomic world indices related to $\mathscr{M}_{4}$ and the second half of Latin alphabet $(l, m, k, \ldots=$ $0,1,2,3)$ to stand for the world indices related to $M_{4}$. The principle foundation of a world-deformation tensor comprises the following two steps [101]: (1) the basis vectors $e_{m}$ at given point $\left(p \in M_{4}\right.$ ) undergo distortion transformations by means of the matrix $\bar{D}$; and (2) the diffeomorphism $x^{\mu}\left(x^{l}\right): M_{4} \rightarrow$ $\mathscr{M}_{4}$ is constructed by seeking a new holonomic coordinates $x^{\mu}\left(x^{l}\right)$ as the solutions of the first-order partial differential equations. Namely,

$$
e_{\mu}=\bar{D}_{\mu}^{l} e_{l}, \quad e_{\mu} \bar{f}_{l}^{\mu}=\bar{\Omega}_{l}^{m} e_{m},
$$

where the conditions of integrability, $\partial_{k} \bar{f}_{l}^{\mu}=\partial_{l} \bar{f}_{k}^{\mu}$, and nondegeneracy, $\|\bar{f}\| \neq 0$, necessarily hold [102, 103]. For reasons that will become clear in the sequel, we write the norm $d s$ of infinitesimal displacement $d x^{\mu}$ on $\mathscr{M}_{4}$ in terms of the spacetime structures of $M_{4}$ :

$$
d s:=e \vartheta=e_{\mu} \otimes \mathcal{\vartheta}^{\mu}=\bar{\Omega}_{l}^{m} e_{m} \otimes \vartheta^{l} \in \mathscr{M}_{4} .
$$

The holonomic metric on the space $\mathscr{M}_{4}$ can be recast in the form $g=g_{\mu \nu} 9^{\mu} \otimes \vartheta^{\nu}=g\left(e_{\mu}, e_{\gamma}\right) \vartheta^{\mu} \otimes \vartheta^{\nu}$, with the components $g_{\mu \nu}=g\left(e_{\mu}, e_{\nu}\right)$ in dual holonomic basis $\left\{\vartheta^{\mu} \equiv d x^{\mu}\right\}$. In order to relate local Lorentz symmetry to more general deformed spacetime, there is, however, a need to introduce the soldering tools, which are the linear frames and forms in tangent fiber bundles to the external general smooth differential manifold, whose components are the so-called tetrad (vierbein) fields. The $\mathscr{M}_{4}$ has at each point a tangent space, $T_{x} \mathscr{M}_{4}$, spanned by the anholonomic orthonormal frame field, $e$, as a shorthand for the collection of the 4-tuplet $\left(e_{0}, \ldots, e_{3}\right)$, where $e_{a}=$ $e_{a}{ }^{\mu} \partial_{\mu}$. We use the first half of Latin alphabet $(a, b, c, \ldots=$ $0,1,2,3)$ to denote the anholonomic indices related to the tangent space. The frame field, $e$, then defines a dual vector, $\vartheta$, of differential forms, $\vartheta=\left(\begin{array}{c}\vartheta^{0} \\ \vdots \\ \vartheta^{3}\end{array}\right)$, as a shorthand for the collection of the $\vartheta^{b}=e^{b}{ }_{\mu} d x^{\mu}$, whose values at every point form the dual basis, such that $\left.e_{a}\right\rfloor \vartheta^{b}=\delta_{a}^{b}$, where $\rfloor$ denotes the interior product; namely, this is a $C^{\infty}$-bilinear map $\rfloor: \Omega^{1} \rightarrow \Omega^{0}$ with $\Omega^{p}$ denoting the $C^{\infty}$-modulo of differential $p$-forms on $\mathscr{M}_{4}$. In components $e_{a}{ }^{\mu} e^{b}{ }_{\mu}=\delta_{a}^{b}$. On the manifold, $\mathscr{M}_{4}$, the tautological tensor field, $i d$, of type $(1,1)$ is defined which assigns to each tangent space the identity linear transformation. Thus for any point $x \in \mathscr{M}_{4}$ and any vector $\xi \in T_{x} \mathscr{M}_{4}$, one has $i d(\xi)=\xi$. In terms of the frame field, the $\vartheta^{a}$ give the expression for id as id := $e \vartheta=e_{0} \otimes \vartheta^{0}+\cdots e_{3} \otimes \vartheta^{3}$, in sense that both sides yield $\xi$ when applied to any tangent vector $\xi$ in the domain of definition of the frame field. One can also consider general transformations of the linear group, $G L(4, R)$, taking any basis into any other set of four linearly independent fields. The notation, $\left\{e_{a}, \vartheta^{b}\right\}$, will be used below for general linear frames.
Let us introduce the so-called first deformation matrices, $\bar{\pi}_{k}^{m}$ and $\pi^{a}{ }_{l}\left(\in G L(4, \mathscr{M}) \forall x^{\mu}\right)$, as follows:

$$
\begin{gathered}
\bar{D}_{\mu}^{m}=e_{\mu}{ }^{k} \bar{\pi}^{m}{ }_{k}, \quad \bar{f}_{l}^{\mu}=e^{\mu}{ }_{k} \bar{\pi}_{l}^{k}, \quad e_{\mu}{ }^{k} e^{\mu}{ }_{m}=\delta_{m}^{k}, \\
\pi_{a}{ }^{m}=e_{a}{ }^{\mu} \bar{D}_{\mu}^{m}, \quad \pi_{l}^{a}=e^{a}{ }_{\mu} \bar{f}_{l}^{\mu},
\end{gathered}
$$

where $g_{\mu \nu} e_{k}^{\mu} e_{s}^{\nu}=\eta_{k s}$ and $\eta_{k s}$ is the metric on $M_{4}$. With this provision, we build up a world-deformation tensor, $\bar{\Omega}^{m}{ }_{l}=$ $\bar{\pi}^{m}{ }_{k} \bar{\pi}_{l}^{k}=\pi_{a}^{m}{ }_{a}^{a}{ }_{l}$, which yields local tetrad deformations

$$
\begin{array}{ll}
e_{a}=\pi_{a}{ }^{m} e_{m}, & \vartheta^{a}=\pi^{a}{ }_{l} \vartheta^{l}, \\
\bar{e}_{k}=\bar{\pi}^{m}{ }_{k} e_{m}, & \bar{\vartheta}^{k}=\bar{\pi}^{k}{ }_{l} \vartheta^{l},
\end{array}
$$

and $d s=i d=e_{a} \otimes \mathcal{V}^{a}=\bar{e}_{k} \otimes \bar{\vartheta}^{k} \in \mathscr{M}_{4}$. The first deformation matrices $\pi$, in general, are the elements of the quotient group $G L(4, \mathscr{M}) / S O(3,1)$. Here $G L(4, \mathscr{M}) / S O(3,1)$ is the manifold of left cosets of the group $G L(4, \mathscr{M}) / S O(3,1)$ over its subgroup $S O(3,1)$. If we deform the cotetrad according to (4), we have two choices to recast metric as follows: either writing the deformation of the metric in the space of tetrads or deforming the tetrad field:

$$
g=o_{a b} \vartheta^{a} \otimes \vartheta^{b}=o_{a b} \pi^{a}{ }_{l} \pi^{b}{ }_{m} \vartheta^{l} \otimes \vartheta^{m}=\gamma_{l m} \vartheta^{l} \otimes \vartheta^{m},
$$

where $\gamma_{l m}$, following [82], is called second deformation matrix and reads $\gamma_{l m}=o_{a b} \pi^{a}{ }_{l} \pi^{b}{ }_{m}$. The deformed metric splits as

$$
g_{\mu \nu}=\Upsilon^{2} \eta_{\mu \nu}+\gamma_{\mu \nu}
$$

where $\Upsilon=\pi^{a}{ }_{a}=\bar{\pi}^{k}{ }_{k}$, and

$$
\gamma_{\mu \nu}=\left(\gamma_{a l}-\Upsilon^{2} o_{a l}\right) e_{\mu}^{a} e_{\nu}^{l}=\left(\gamma_{k s}-\Upsilon^{2} \eta_{k s}\right) e_{\mu}^{k} e_{\nu}^{s} .
$$

The anholonomic orthonormal frame field, $e$, relates $g$ to the tangent space metric, $o_{a b}=\operatorname{diag}(+---)$, by $o_{a b}=g\left(e_{a}, e_{b}\right)=$ $g_{\mu \nu} e_{a}{ }^{\mu} e_{b}{ }^{\nu}$, which has the converse $g_{\mu \nu}=o_{a b} e^{a}{ }_{\mu} e^{b}{ }_{\nu}$ because $e_{a}^{\mu} e^{a}{ }_{\nu}=\delta_{\nu}^{\mu}$. The $\gamma_{l m}$ can be decomposed in terms of symmetric, $\pi_{(a l)}$, and antisymmetric, $\pi_{[a l]}$, parts of the matrix $\pi_{a l}=o_{a c} \pi^{c}{ }_{l}$ (or, respectively, in terms of $\bar{\pi}_{(k l)}$ and $\bar{\pi}_{[k l]}$, where $\left.\bar{\pi}_{k l}=\eta_{k s} \bar{\pi}_{l}^{s}\right)$ as

$$
\begin{aligned}
\gamma_{a l}= & \Upsilon^{2} o_{a l}+2 \curlyvee \Theta_{a l}+o_{c d} \Theta^{c}{ }_{a} \Theta^{d}{ }_{l} \\
& +o_{c d}\left(\Theta^{c}{ }_{a} \varphi^{d}{ }_{l}+\varphi^{c}{ }_{a} \Theta^{d}{ }_{l}\right)+o_{c d} \varphi^{c}{ }_{a} \varphi^{d}{ }_{l},
\end{aligned}
$$

where

$$
\pi_{a l}=\Upsilon_{a l}+\Theta_{a l}+\varphi_{a l},
$$

provided $\Theta_{a l}$ is the traceless symmetric part and $\varphi_{a l}$ is the skew symmetric part of the first deformation matrix. The anholonomy objects defined on the tangent space, $T_{x} \mathscr{M}_{4}$, read

$$
C^{a}:=d \vartheta^{a}=\frac{1}{2} C^{a}{ }_{b c} \vartheta^{b} \wedge \vartheta^{c},
$$


where the anholonomy coefficients, $C^{a}{ }_{b c}$, which represent the curls of the base members, are

$$
\begin{aligned}
C_{a b}^{c} & =-\vartheta^{c}\left(\left[e_{a}, e_{b}\right]\right)=e_{a}{ }^{\mu} e_{b}{ }^{\nu}\left(\partial_{\mu} e^{c}{ }_{\nu}-\partial_{\nu} e^{c}{ }_{\mu}\right) \\
& =-e^{c}{ }_{\mu}\left[e_{a}\left(e_{b}{ }^{\mu}\right)-e_{b}\left(e_{a}^{\mu}\right)\right] \\
& =2 \pi^{c}{ }_{l} e_{m}^{\mu}\left(\pi^{-1^{m}}\left[\partial_{\mu} \pi^{-1^{l}}{ }_{b]}\right) .\right.
\end{aligned}
$$

In particular case of constant metric in the tetradic space, the deformed connection can be written as

$$
\Gamma_{b c}^{a}=\frac{1}{2}\left(C_{b c}^{a}-o^{a a^{\prime}} o_{b b^{\prime}} C_{a^{b^{\prime} c}}^{b^{\prime}}-o^{a a^{\prime}} o_{c c^{\prime}} C_{a^{\prime} b}^{c^{\prime}}\right) .
$$

The deformation $\bar{\Omega}: M_{4} \rightarrow \mathscr{M}_{4}$ comprises the following two $4 \mathrm{D}$ deformations $\breve{\Omega}: M_{4} \rightarrow V_{4}$ and $\Omega: V_{4} \rightarrow \mathscr{M}_{4}$, where $V_{4}$ is the semi-Riemannian space and $\breve{\Omega}$ and $\Omega$ are the corresponding world-deformation tensors. All magnitudes related to the space, $V_{4}$, will be denoted with an over " ". According to (1), now we have $\breve{\Omega}^{m}{ }_{l}=\breve{D}_{\mu}^{m} \breve{f}_{l}^{\mu}$ and $\Omega^{\mu}{ }_{\nu}=D_{\rho}^{\mu} f_{\nu}^{\rho}$, provided

$$
\begin{array}{ll}
\breve{e}_{\mu}=\breve{D}_{\mu}^{l} e_{l}, & \breve{e}_{\mu} \breve{f}_{l}^{\mu}=\breve{\Omega}_{l}^{m} e_{m}, \\
e_{\rho}=D_{\rho}^{\mu} \breve{e}_{\mu}, & e_{\rho} f_{\nu}^{\rho}=\Omega^{\mu}{ }_{\nu} \breve{e}_{\mu} .
\end{array}
$$

In analogy with (3), the following relations hold:

$$
\begin{gathered}
\breve{D}_{\mu}^{m}=\breve{e}_{\mu}{ }^{k} \breve{\pi}^{m}{ }^{m}, \quad \breve{f}_{l}^{\mu}=\breve{e}^{\mu}{ }_{k} \breve{\pi}^{k}{ }_{l}, \quad \breve{e}_{\mu}{ }^{k} \breve{e}^{\mu}{ }_{m}=\delta_{m}^{k}, \\
\breve{\pi}^{k}{ }^{m}=\breve{e}_{a}{ }^{\mu} \breve{D}_{\mu}^{m}, \quad \breve{\pi}^{a}{ }_{l}=\breve{e}^{a}{ }_{\mu} \breve{f}_{l}^{\mu},
\end{gathered}
$$

where $\breve{\Omega}^{m}{ }_{l}=\breve{\pi}^{m}{ }_{\rho} \breve{\pi}^{\rho}{ }_{l}, \Omega^{\mu}{ }_{v}=\pi^{\mu}{ }_{\rho} \pi^{\rho}{ }_{v}$. So, $\breve{g}_{\mu \nu} \breve{e}_{k}{ }^{\mu} \breve{e}_{s}{ }^{\nu}=\eta_{k s}$, and

$$
\begin{gathered}
D_{\rho}^{\mu}=e_{\nu}{ }^{\mu} \pi_{\rho}^{\nu}, \quad f_{\nu}^{\rho}=e_{\mu}^{\rho} \pi_{\nu}^{\mu}, \quad e_{\nu}^{\mu} e_{\rho}^{\nu}=\delta_{\rho}^{\mu}, \\
\pi_{a}{ }^{\mu}=e_{a}^{\rho} D_{\rho}^{\mu}, \quad \pi^{a}{ }_{\nu}=e_{\rho}^{a} f_{\nu}^{\rho} .
\end{gathered}
$$

The norm $d \breve{s}=i \breve{d}$ of the infinitesimal displacement $d \breve{x}^{\mu}$ on the $V_{4}$ can be written in terms of the spacetime structures of $M_{4}$ as

$$
i \breve{d}:=\breve{e} \breve{\vartheta}=\breve{\Omega}^{m} e_{m} \otimes \vartheta^{l} \in V_{4} .
$$

The holonomic metric can be recast in the form

$$
\breve{g}=\breve{g}_{\mu \nu} \breve{\vartheta}^{\mu} \otimes \breve{\vartheta}^{\nu}=\breve{g}\left(\breve{e}_{\mu}, \breve{e}_{\nu}\right) \breve{\vartheta}^{\mu} \otimes \breve{\vartheta}^{\nu}
$$

The anholonomy objects defined on the tangent space, $\breve{T}_{\breve{x}} V_{4}$, read

$$
\breve{C}^{a}:=d \breve{\vartheta}^{a}=\frac{1}{2} \breve{C}^{a}{ }_{b c} \breve{\vartheta}^{b} \wedge \breve{\vartheta}^{c},
$$

where the anholonomy coefficients, $\breve{C}^{a}{ }_{b c}$, which represent the curls of the base members, are

$$
\begin{aligned}
\breve{C}_{a b}^{c} & =-\breve{\vartheta}^{c}\left(\left[\breve{e}_{a}, \breve{e}_{b}\right]\right)=\breve{e}_{a}{ }^{\mu} \breve{e}_{b}{ }^{\nu}\left(\breve{\partial}_{\mu} \breve{e}_{\nu}^{c}{ }_{\nu}-\breve{\partial}_{\nu} \breve{e}_{\mu}^{c}\right) \\
& =-\breve{e}_{\mu}^{c}\left[\breve{e}_{a}\left(\breve{e}_{b}{ }^{\mu}\right)-\breve{e}_{b}\left(\breve{e}_{a}{ }^{\mu}\right)\right] .
\end{aligned}
$$

The connection form in terms of the frame field is then determined by

$$
d \breve{\vartheta}+\breve{\Gamma} \wedge \breve{\vartheta}=0,
$$

and the curvature by

$$
\breve{R}=d \breve{\Gamma}+\breve{\Gamma} \wedge \breve{\Gamma},
$$

where the (anholonomic) Levi-Civita (or Christoffel) connection can be written as

$$
\left.\left.\left.\breve{\Gamma}_{a b}:=\breve{e}_{[a}\right\rfloor d \breve{\vartheta}_{b]}-\frac{1}{2}\left(\breve{e}_{a}\right\rfloor \breve{e}_{b}\right\rfloor d \breve{\vartheta}_{c}\right) \wedge \breve{\vartheta}^{c}
$$

and $\breve{\vartheta}_{c}$ is understood as the downindexed 1-form $\breve{\vartheta}_{c}=o_{c b} \breve{\vartheta}^{b}$. In the usual Riemannian language involving the holonomic metric $\breve{g}$ (17) defined from the tetrads, the anholonomic Christoffel connection (22) transforms into

$$
\breve{\Gamma}_{a b}:=-d \breve{x}^{\mu} \breve{e}_{[a}^{\nu}\left(\partial_{\mu} \breve{e}_{v b]}-\breve{\Gamma}_{\mu \nu}^{\sigma} \breve{e}_{\sigma b]}\right),
$$

where $\breve{\Gamma}_{\mu \nu}^{\sigma}$ is the ordinary holonomic Levi-Civita connection. The corresponding curvature reduces to

$$
\breve{R}_{a b}=\frac{1}{2} d \breve{x}^{\mu} \wedge d \breve{x}^{\nu} \breve{e}_{[a}{ }^{\rho} \breve{e}_{\sigma b]} \breve{R}_{\mu \nu \rho}{ }^{\sigma},
$$

provided $\breve{R}_{\mu \nu \rho}{ }^{\sigma}:=\breve{\vartheta}^{\sigma}\left(R_{\breve{e}_{v} \breve{\rho}_{\rho}} \breve{e}_{\mu}\right)$ is the ordinary Riemann tensor:

$$
\breve{R}_{\mu \nu \rho}^{\sigma}=2\left(\breve{\partial}_{[\mu} \breve{\Gamma}_{\nu] \rho}^{\sigma}+\breve{\Gamma}_{[\mu \lambda}^{\sigma} \breve{\Gamma}_{\nu] \rho}^{\lambda}\right) \text {, }
$$

and hence, the Ricci tensor takes the form

$$
\breve{R}=\breve{R}_{\breve{e}_{\mu} \breve{e}_{\nu}} \breve{\vartheta}^{\mu} \breve{\vartheta}^{\nu}=\breve{R}_{\mu \nu} \breve{\vartheta}^{\mu} \breve{\vartheta}^{\nu}=\breve{R}_{\mu \sigma \nu}^{\sigma} \breve{\vartheta}^{\mu} \breve{\vartheta}^{\nu}
$$

The norm id (2) can then be written in terms of the spacetime structures of $V_{4}$ and $M_{4}$ :

$$
\begin{aligned}
i d & :=e \vartheta=e_{\rho} \otimes \vartheta^{\rho}=e_{a} \otimes \vartheta^{a}=\Omega^{\mu}{ }_{\nu} \breve{e}_{\mu} \otimes \breve{\vartheta}^{\nu} \\
& =\Omega^{a}{ }_{b} e_{a} \vartheta^{b}=\bar{\Omega}_{l}^{m} e_{m} \otimes \vartheta^{l} \in \mathscr{M}_{4},
\end{aligned}
$$

provided

$$
\begin{array}{ccc}
\Omega^{a}{ }_{b}=\pi^{a}{ }_{c} \pi^{c}{ }_{b}=\Omega^{\mu}{ }_{\nu} \breve{e}^{a}{ }_{\mu} \breve{e}_{b}{ }^{\nu}, & e_{\rho}=\pi^{\nu}{ }_{\rho} \breve{e}_{v}, \\
\vartheta^{\rho}=\pi_{\mu}{ }^{\rho} \breve{\vartheta}^{\mu}, & e_{c}=\pi_{c}{ }^{a} \breve{e}_{a}, & \vartheta^{c}=\pi_{b}^{c}{ }_{b} \breve{\vartheta}^{b} .
\end{array}
$$

Under a local tetrad deformation (28), a general spin connection transforms according to

$$
\omega_{b \mu}^{a}=\pi_{c}^{a} \breve{\omega}_{d \mu}^{c} \pi_{b}^{d}+\pi_{c}^{a} \partial_{\mu} \pi_{b}^{c}=\pi_{l}{ }^{a} \partial_{\mu} \pi_{b}^{l} .
$$

We have then two choices to recast metric as follows:

$$
g=o_{a b} \vartheta^{a} \otimes \vartheta^{b}=o_{a b} \pi^{a}{ }_{c} \pi^{b}{ }_{d} \breve{\vartheta}^{c} \otimes \breve{\vartheta}^{d}=\gamma_{c d} \breve{\vartheta}^{c} \otimes \breve{\vartheta}^{d} .
$$

In the first case, the contribution of the Christoffel symbols constructed by the metric $\gamma_{a b}=o_{c d} \pi^{c}{ }_{a} \pi^{d}{ }_{b}$ reads

$$
\begin{aligned}
\Gamma_{b c}^{a}= & \frac{1}{2}\left(\breve{C}_{b c}^{a}-\gamma^{a a^{\prime}} \gamma_{b b^{\prime}} \breve{C}_{a^{\prime} c}-\gamma^{a a^{\prime}} \gamma_{c c^{\prime}} \breve{C}_{a^{\prime} b}\right) \\
& \left.\left.\left.+\frac{1}{2} \gamma^{a a^{\prime}}\left(\breve{e}_{c}\right\rfloor d \gamma_{b a^{\prime}}-\breve{e}_{b}\right\rfloor d \gamma_{c a^{\prime}}-\breve{e}_{a^{\prime}}\right\rfloor d \gamma_{b c}\right) .
\end{aligned}
$$


As before, the second deformation matrix, $\gamma_{a b}$, can be decomposed in terms of symmetric, $\pi_{(a b)}$, and antisymmetric, $\pi_{[a b]}$, parts of the matrix $\pi_{a b}=o_{a c} \pi_{b}^{c}$. So,

$$
\pi_{a b}=\Upsilon_{a b}+\Theta_{a b}+\varphi_{a b}
$$

where $\Upsilon=\pi^{a}{ }_{a}, \Theta_{a b}$ is the traceless symmetric part, and $\varphi_{a b}$ is the skew symmetric part of the first deformation matrix. In analogy with (6), the deformed metric can then be split as

$$
g_{\mu \nu}(\pi)=\Upsilon^{2}(\pi) \breve{g}_{\mu \nu}+\gamma_{\mu \nu}(\pi),
$$

where

$$
\gamma_{\mu \nu}(\pi)=\left[\gamma_{a b}-\Upsilon^{2} o_{a b}\right] \breve{e}_{\mu}^{a} \breve{e}_{\nu}^{b} .
$$

The inverse deformed metric reads

$$
g^{\mu \nu}(\pi)=o^{c d} \pi^{-1^{a}} \pi_{d}^{-1^{b}} \breve{e}_{a}^{\mu} \breve{e}_{b}^{\nu},
$$

where $\pi^{-1}{ }_{c}^{a} \pi^{c}{ }_{b}=\pi^{c}{ }_{b} \pi^{-1^{a}}{ }_{c}=\delta_{b}^{a}$. Hence, the usual LeviCivita connection is related to the original connection by the relation

$$
\widetilde{\Gamma}_{\rho \sigma}^{\mu}=\breve{\Gamma}_{\rho \sigma}^{\mu}+\Pi_{\rho \sigma}^{\mu},
$$

provided

$$
\begin{aligned}
\Pi_{\rho \sigma}^{\mu}= & 2 g^{\mu \nu} \breve{g}_{\nu(\rho} \breve{\nabla}_{\sigma)} \breve{\Upsilon}-\breve{g}_{\rho \sigma} g^{\mu \nu} \breve{\nabla}_{\nu} \breve{\Upsilon} \\
& +\frac{1}{2} g^{\mu \nu}\left(\breve{\nabla}_{\rho} \breve{\gamma}_{\nu \sigma}+\breve{\nabla}_{\sigma} \breve{\gamma}_{\rho \nu}-\breve{\nabla}_{\nu} \breve{\gamma}_{\rho \sigma}\right),
\end{aligned}
$$

where $\breve{\nabla}$ is the covariant derivative. The contravariant deformed metric $g^{\nu \rho}$ is defined as the inverse of $g_{\mu \nu}$ : $g_{\mu \nu} g^{\nu \rho}=\delta_{\mu}^{\rho}$. Hence, the connection deformation $\Pi_{\rho \sigma}^{\mu}$ acts like a force that deviates the test particles from the geodesic motion in the space, $V_{4}$. A metric-affine space $\left(\mathscr{M}_{4}, g, \Gamma\right)$ is defined to have a metric and a linear connection that need not be dependent on each other. In general, the lifting of the constraints of metric compatibility and symmetry yields the new geometrical property of the spacetime, which are the nonmetricity 1-form $N_{a b}$ and the affine torsion 2-form $T^{a}$ representing a translational misfit (for a comprehensive discussion see $[35-37,94])$. These, together with the curvature 2 -form $R_{a}{ }^{b}$, symbolically can be presented as $[8,9]$

$$
\left(N_{a b}, T^{a}, R_{a}^{b}\right) \sim \mathscr{D}\left(g_{a b}, \vartheta^{a}, \Gamma_{a}^{b}\right),
$$

where $\mathscr{D}$ is the covariant exterior derivative. If the nonmetricity tensor $N_{\lambda \mu \nu}=-\mathscr{D}_{\lambda} g_{\mu \nu} \equiv-g_{\mu \nu ; \lambda}$ does not vanish, the general formula for the affine connection written in the spacetime components is

$$
\Gamma_{\mu \nu}^{\rho}=\breve{\Gamma}_{\mu \nu}^{\rho}+K_{\mu \nu}^{\rho}-N_{\mu \nu}^{\rho}+\frac{1}{2} N_{(\mu \nu)}^{\rho},
$$

where $\breve{\Gamma}_{\mu \nu}^{\rho}$ is the Riemann part and $K_{\mu \nu}^{\rho}:=2 Q_{(\mu \nu)}^{\rho}+Q_{\mu \nu}^{\rho}$ is the non-Riemann part-the affine contortion tensor. The torsion, $Q_{\mu \nu}^{\rho}=(1 / 2) T_{\mu \nu}^{\rho}=\Gamma_{[\mu \nu]}^{\rho}$ given with respect to a holonomic frame, $d \vartheta^{\rho}=0$, is a third-rank tensor, antisymmetric in the first two indices, with 24 independent components. In the presence of curvature and torsion, the coupling prescription of a general field carrying an arbitrary representation of the Lorentz group will be

$$
\partial_{\mu} \longrightarrow \mathscr{D}_{\mu}=\partial_{\mu}-\frac{i}{2}\left(\omega_{\mu}^{a b}-K_{\mu}^{a b}\right) J_{a b}
$$

with $J_{a b}$ denoting the corresponding Lorentz generator. The Riemann-Cartan manifold, $U_{4}$, is a particular case of the general metric-affine manifold $\mathscr{M}_{4}$, restricted by the metricity condition $N_{\lambda \mu \nu}=0$, when a nonsymmetric linear connection is said to be metric compatible. The Lorentz and diffeomorphism invariant scalar curvature, $R$, becomes either a function of $e^{a}{ }_{\mu}$ only or $g_{\mu \nu}$ :

$$
R(\omega) \equiv e_{a}^{\mu} e_{b}{ }^{\nu} R_{\mu \nu}^{a b}(\omega)=R(g, \Gamma) \equiv g^{\rho v} R_{\rho \mu \nu}^{\mu}(\Gamma)
$$

2.1. Determination of $\bar{D}$ and $\bar{f}$. Let $\omega^{a b}=\omega_{\mu}^{a b} \wedge d x^{\mu}$ be the $1-$ forms of corresponding connections assuming values in the Lorentz Lie algebra. The action for gravitational field can be written in the form

$$
S_{g}=\breve{S}+S_{Q}
$$

where the integral,

$$
\begin{aligned}
\breve{S} & =-\frac{1}{4 æ} \int \star \breve{R}=-\frac{1}{4 æ} \int \star \breve{R}_{c d} \wedge \vartheta^{c} \wedge \vartheta^{d} \\
& =-\frac{1}{2 æ} \int \breve{R} \sqrt{-g} d \Omega,
\end{aligned}
$$

is the usual Einstein action, with the coupling constant relating to the Newton gravitational constant $æ=8 \pi G_{N} / c^{4}, S_{\mathrm{Q}}$ is the phenomenological action of the spin-torsion interaction, and $\star$ denotes the Hodge dual (Appendix B). The variation of the connection 1-form $\omega^{a b}$ yields

$$
\delta S_{\mathrm{Q}}=\frac{1}{æ} \int \star \mathscr{T}_{a b} \wedge \delta \omega^{a b},
$$

where

$$
\begin{aligned}
\star \mathscr{T}_{a b} & :=\frac{1}{2} \star\left(Q_{a} \wedge e_{b}\right)=Q^{c} \wedge \vartheta^{d} \varepsilon_{c d a b} \\
& =\frac{1}{2} Q^{c}{ }_{\mu \nu} \wedge e^{d}{ }_{\alpha} \varepsilon_{a b c d} \vartheta^{\mu \nu \alpha}
\end{aligned}
$$

here we used the abbreviated notations for the wedge product monomials, $\vartheta^{\mu \nu \alpha \cdots}=\vartheta^{\mu} \wedge \vartheta^{\nu} \wedge \vartheta^{\alpha} \wedge \cdots$, defined on the $U_{4}$ space and

$$
Q^{a}=D \vartheta^{a}=d \vartheta^{a}+\omega^{a}{ }_{b} \wedge \vartheta^{b}
$$

The variation of the action describing the macroscopic matter sources $S_{m}$ with respect to the coframe $\vartheta^{a}$ and connection 1form $\omega^{a b}$ reads

$$
\delta S_{m}=\int \delta L_{m}=\int\left(-\star \theta_{a} \wedge \delta \vartheta^{a}+\frac{1}{2} \star \Sigma_{a b} \wedge \delta \omega^{a b}\right),
$$


where $\star \theta_{a}$ is the dual 3 -form relating to the canonical energymomentum tensor, $\theta_{a}^{\mu}$, by

$$
\star \theta_{a}=\frac{1}{3 !} \theta_{a}^{\mu} \varepsilon_{\mu \nu \alpha \beta} \vartheta^{\nu \alpha \beta} .
$$

And $\star \Sigma_{a b}=-\star \Sigma_{b a}$ is the dual 3-form corresponding to the canonical spin tensor, which is identical with the dynamical spin tensor $S_{a b c}$, namely,

$$
\star \Sigma_{a b}=S_{a b}^{\mu} \varepsilon_{\mu \nu \alpha \beta} \gamma^{\gamma \alpha \beta} .
$$

The variation of the total action, $S=S_{g}+S_{m}$, with respect to the $e_{a}, \omega^{a b}$, and $\Phi$, gives the field equations as follows:

$$
\begin{aligned}
& \text { (1) } \frac{1}{2} \breve{R}_{c d} \wedge \vartheta^{c}=æ \theta_{d}=0, \\
& \text { (2) } \star \mathscr{T}_{a b}=-\frac{1}{2} æ \star \Sigma_{a b}, \\
& \text { (3) } \frac{\delta L_{m}}{\delta \Phi}=0, \quad \frac{\delta L_{m}}{\delta \bar{\Phi}}=0 .
\end{aligned}
$$

The DC-members $\bar{D}$ and $\bar{f}$ can then be determined as follows:

$$
\bar{D}_{a}^{l}=\eta^{l m}\left\langle e_{a}, e_{m}\right\rangle, \quad \bar{f}_{l}^{a}=\eta_{l m} \vartheta^{a}\left(\vartheta^{-1}\right)^{m} .
$$

However, it should be emphasized that the standard Riemannian (and its extensions) space interacting quantum field theory cannot be a satisfactory ground for addressing the most important processes of rearrangement of vacuum state and gauge symmetry breaking in gravity at huge energies. The difficulties here arise because the Riemannian geometry, in general, does not admit a group of isometries and it is impossible to define energy-momentum as Noether local currents related to exact symmetries. This, in turn, posed severe problem of nonuniqueness of the physical vacuum and the associated Fock space. A definition of positive frequency modes cannot, in general, be unambiguously fixed in the past and future, which leads to $\mid$ in $\rangle \neq \mid$ out $\rangle$, because the state $\mid$ in $\rangle$ is unstable against decay into many particle |out $\rangle$ states due to interaction processes allowed by lack of Poincaré invariance. A nontrivial Bogolubov transformation between past and future positive frequency modes implies that particles are created from the vacuum and this is one of the reasons for $\mid$ in $\rangle \neq \mid$ out $\rangle$. So, it is another line of reasoning which supports the side suggested in this section approach. Namely, the actual advantage of our approach compared to the more conservative direct constructions, using the vierbeins, metric, and various connections, is that it allows various generalizations and alternatives of the standard gravity. In this framework, in particular, we develop an alternative distortion gauge induced fiber-bundle formulation of gravitation-General Gauge Principle (GGP) [101]. In this, we restrict ourself to consider only the simplest spacetime deformation map, $\breve{\Omega}: M_{4} \rightarrow V_{4}\left(\Omega^{\mu}{ }_{v} \equiv \delta_{v}^{\mu}\right)$. Whereas given the principal fiber bundle $\breve{P}\left(V_{4}, G_{V} ; \breve{s}\right)$ with the structure group $G_{V}$, the local coordinates $\breve{p} \in \breve{P}$ are $\breve{p}=$ $\left(\breve{x}, U_{V}\right)$, where $\breve{x} \in V_{4}$ and $U_{V} \in G_{V}$, the total bundle space
$\breve{P}$ is a smooth manifold and the surjection $\breve{s}$ is a smooth map $\breve{s}: \breve{P} \rightarrow V_{4}$. The collection of matter fields of arbitrary spins $\breve{\Phi}(\breve{x})$ takes values in standard fiber over $\breve{x}: \breve{s}^{-1}\left(\breve{U}_{i}\right)=\breve{U}_{i} \times \breve{F}_{\breve{x}}$ where $\left\{\breve{U}_{i}\right\}$ is a set of open coverings of $V_{4}$. The action of the structure group $G_{V}$ on $\breve{P}$ defines an isomorphism of the Lie algebra $\breve{\mathfrak{g}}$ of $G_{V}$ onto the Lie algebra of vertical vector fields on $\breve{P}$ tangent to the fiber at each $\breve{p} \in \breve{P}$ called fundamental. Note that an invariance of the Lagrangian $L_{\breve{\Phi}}$ under spacetime diffeomorphisms in $V_{4}$ implies an invariance of $L_{\breve{\Phi}}$ under the $G_{V}$ group and vice versa if, and only if, the generalized local gauge transformations of the fields $\breve{\Phi}(\breve{x})$ and their covariant derivative $\breve{\nabla}_{\mu} \breve{\Phi}(\breve{x})$ are introduced by finite local $U_{V}$ gauge transformations:

$$
\begin{aligned}
\breve{\Phi}^{\prime}(\breve{x}) & =U_{V}(\breve{x}) \breve{\Phi}(\breve{x}), \\
{\left[\breve{\gamma}^{\mu}(\breve{x}) \breve{\nabla}_{\mu} \breve{\Phi}(\breve{x})\right]^{\prime} } & =U_{V}(\breve{x})\left[\breve{\gamma}^{\mu}(\breve{x}) \breve{\nabla}_{\mu} \breve{\Phi}(\breve{x})\right] .
\end{aligned}
$$

Here $\breve{\nabla}_{\mu}$ denotes the covariant derivative agreed with the metric $\breve{g}^{\mu \nu}=(1 / 2)\left(\breve{\gamma}^{\mu} \breve{\gamma}^{\nu}+\breve{\gamma}^{\nu} \breve{\gamma}^{\mu}\right)$. For example, $\widetilde{\gamma}^{\mu}(\widetilde{x})=$ $\tilde{e}_{a}{ }^{\mu}(\widetilde{x}) \gamma^{a}$ for the spinor field $(j=1 / 2)$, where $\gamma^{a}$ are the Dirac matrices, $\breve{\nabla}_{\mu}=\breve{\partial}_{\mu}+\breve{\Gamma}_{\mu}, \breve{\Gamma}_{\mu}(\breve{x})=(1 / 2) J^{a b} \breve{e}_{a}{ }^{\nu}(\breve{x}) \breve{\partial}_{\mu} \breve{e}_{b v}(\breve{x})$, and $J^{a b}=-(1 / 4)\left[\gamma^{a}, \gamma^{b}\right]$. To involve a drastic revision of the role of gauge fields in the physical concept of the spacetime deformation/distortion, we generalize the standard gauge scheme by exploring a new special type of distortion gauge field, $(a)\left(\equiv a_{m}\left(x^{l}\right), x^{l} \in M_{4}\right)$, which is assumed to act on the external spacetime groups. That is, we also consider the principle fiber bundle, $P\left(M_{4}, U^{\text {loc }} ; s\right)$, with the base space $M_{4}$, the structure group $U^{\text {loc }}$, and the surjection $s$. The matter fields $\Phi(x)$ take values in the standard fiber which is the Hilbert vector space where a linear representation $U(x)$ of group $U^{\text {loc }}$ is given. This space can be regarded as the Lie algebra of the group $U^{\text {loc }}$ upon which the Lie algebra acts according to the law of the adjoint representation: $a \leftrightarrow$ ada $\Phi \rightarrow[a, \Phi]$. The GGP framework accounts for the gravitation gauge group $G_{V}$ generated by the hidden local internal symmetry $U^{\text {loc }}$. We pursue a principle goal to build up the world-deformation tensor, $\breve{\Omega}(F)=\breve{D}(a) \breve{f}(a)$, where $F$ is the differential form of gauge field $F=(1 / 2) F_{n m} \vartheta^{n} \wedge$ $9^{m}$. We connect the structure group $G_{V}$, further, to the nonlinear realization of the Lie group $G_{D}$ of distortion of the $M_{4}$. The nonlinear realization technique or the method of phenomenological Lagrangians [104-110] provides a way to determine the transformation properties of fields defined on the quotient space. In accord, we treat the distortion group $G_{D}$ and its stationary subgroup $H=S O(3)$, respectively, as the dynamical group and its algebraic subgroup. The fundamental field is distortion gauge field (a) and, thus, all the fundamental gravitational structures in fact, the metric as much as the coframes and connections, acquire a distortion-gauge induced theoretical interpretation. We derive the Maurer-Cartan structure equations, where the distortion fields (a) are treated as the Goldstone fields. This framework explores the most important processes of rearrangement of vacuum state and a spontaneous breaking of gravitation gauge symmetry at huge energies. 
2.2. TSSD: Revisited. For the benefit of the reader, a brief outline of the key ideas behind the TSSD [82], as a guiding principle, is given in this section to make the rest of the paper understandable. Before we report on the physical foundation of post-Riemannian geometry, we may remark on the form of generic spacetime deformation, $\pi(x)$. When torsion is nonvanishing, the affine connection is no longer coincident with the Levi-Civita connection, and the geometry is no longer Riemannian, but one has a RC spacetime, $U_{4}$, with a nonsymmetric, but metric-compatible, connection. Teleparallel gravity, in turn, represented a new way of including torsion into general relativity, an alternative to the scheme provided by the usual Einstein-Cartan-SciamaKibble approach. However, the gravitational coupling of the fundamental fields in teleparallel gravity is a very controversial subject $[15,111-114]$. The basic difficulty lies in the definition of the spin connection and consequently in the correct form of the gravitational coupling prescription. For a specific choice of the free parameters, teleparallel gravity becomes completely equivalent to GR. In this case it is usually referred to as the teleparallel equivalent of GR. From this point of view, curvature and torsion are simply alternative ways of describing the gravitational field and are consequently related to the same degrees of freedom of gravity. Teleparallel gravity attributes gravitation to torsion, but in this case torsion accounts for gravitation not by geometrizing the interaction, but by acting as a force. The fundamental difference between these two theories above was that, whereas in the former a torsion is a propagating field having as a source-the energy-momentum tensor, in the latter a torsion is not a propagating field having as a source-the total spin, a point which can be considered a drawback of this model. In fact, the two physical interpretations of torsion described above are clearly conflictive. This problem can be solved just only by experiment. Therefore, we have to separate, from the very outset, these two completely different cases. This reasoning supports our choice of a double deformation map. Namely, following [82], we assume that the spacetime deformation $\pi(x)$ comprises the two ingredient deformations $(\dot{\pi}(\dot{x}), \sigma(x))$. Hence, local tetrad deformations $(\breve{e}, \breve{\vartheta}) \rightarrow(e, \vartheta)(13)$ are performed according to the following heuristic map, in two steps (two-step deformation map):

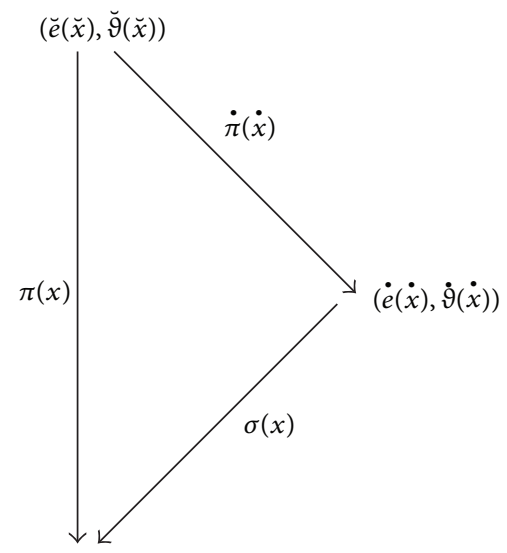

$(e(x), \vartheta(x))$
Thereby the first deformation proper matrix, $\dot{\pi}(\dot{x}):=\left(\dot{\pi}_{b}{ }^{a}\right)(\dot{x})\left(\left|\dot{\pi}_{b}{ }^{a}\right|=1\right)$, satisfies the following peculiar condition:

$$
\dot{\pi}_{c}^{a}(\dot{x}) \dot{\partial}_{\mu} \dot{\pi}_{b}^{-1 c}(\dot{x})=\breve{\omega}_{b \mu}^{a}(\breve{x})
$$

where $\breve{\omega}_{b \mu}^{a}(\breve{x})$ is a $S O(3,1)$ valued Lorentz (i.e., traceless with respect to its indices $a$ and $b$ ) spin connection defined on the semi-Riemannian space. Under a local spacetime deformation $\dot{\pi}(\dot{x})$, the tetrad changes according to

$$
\begin{gathered}
\dot{e}_{c}=\dot{\pi}_{c}{ }^{a} \breve{e}_{a}, \quad \dot{\vartheta}^{c}=\dot{\pi}^{c}{ }_{b} \breve{\vartheta}^{b}, \\
\dot{e}_{a} \otimes \dot{\vartheta}^{a}=\dot{\Omega}^{a}{ }_{b} \breve{e}_{a} \otimes \breve{\vartheta}^{b} .
\end{gathered}
$$

A particular solution to (53) is then (see Appendix A)

$$
\dot{\pi}(\dot{x})=\dot{\pi}(0) \exp \left[-\int_{0}^{\dot{x}} \breve{\omega}_{\mu}(\breve{x}) d \dot{x}^{\prime \mu}\right] .
$$

However, a general solution can be obtained by replacing $\dot{\pi}(0) \rightarrow \pi_{B}(\dot{x}) \equiv \dot{\pi}(0) B(\dot{x})$ in expression (55), where $B(\dot{x})$ is any proper matrix with determinant $1(|B(\dot{x})|=1)$. The solution (55) resembles the exponential of a bivector. The bivectors are isomorphic to skew-symmetric matrices which generate orthogonal matrices with determinant 1 through the exponential map. Therefore, bivectors are used to generate rotations in any dimension through the exponential map and are a useful tool for classifying such rotations. Recall that, in the case of spacetime rotations, the geometric algebra is $\mathrm{Cl}_{3,1}(R)$, and the subspace of bivectors is $\wedge 2 R_{3,1}$. Accordingly, the exponential map (55) generates set of all arbitrary rotations (54) of the orthonormal frame $\breve{e}_{a}(\breve{x})$ in tangent space, which form the Lorentz group. On the other hand, the universality of gravitation allows the Levi-Civita connection to be interpreted as part of the spacetime definition. The form of the Riemannian connection (22), which is a function of tetrad fields and their derivatives, shows that the relative orientation of the orthonormal frame $\breve{e}_{a}(\breve{x}+d \breve{x})$ with respect to $\breve{e}_{a}(\breve{x})$ (parallel transported to $(\breve{x}+d \breve{x})$ ) is completely fixed by the metric. Since a change in this orientation is described by Lorentz transformations, it does not induce any gravitational effects; therefore, from the point of view of the principle of equivalence, there is no reason to prevent independent (due to arbitrary deformations (54)) Lorentz rotations of local frames in the space under consideration. If we want to use this freedom, the spin connection should contain a part which is independent of the metric, which will realize an independent Lorentz rotation of frames under parallel transport. In this way, we are led to a description of gravity which is not in semi-Riemannian space. If all inertial frames at a given point are treated on an equal footing, the spacetime has to have torsion, which is the antisymmetric 
part of the affine connection. By virtue of (53) or (55), a general deformed spin connection vanishes:

$$
\begin{aligned}
\dot{\omega}_{b \mu}^{a} & =\dot{\pi}_{c}{ }^{a} \breve{\omega}_{d \mu}^{c} \dot{\pi}_{b}{ }_{b}+\dot{\pi}_{c}{ }^{a} \dot{\partial}_{\mu} \dot{\pi}^{c}{ }_{b} \\
& =\dot{e}^{a}{ }_{\sigma} \dot{\Gamma}_{\rho \mu}^{\sigma} \dot{e}_{b}{ }^{\rho}+\dot{e}^{a}{ }_{\rho} \dot{\partial}_{\mu} \dot{e}_{b}{ }^{\rho} \equiv 0
\end{aligned}
$$

In fact, a general linear connection, $\dot{\Gamma}_{\rho \sigma}^{\mu}$, is related to the corresponding spin connection, $\dot{\omega}_{b \mu}^{a}$, through the inverse

$$
\dot{\Gamma}_{\rho \sigma}^{\mu}=\dot{e}_{a}^{\mu} \dot{\partial}_{\sigma} \dot{e}_{\rho}^{a}+\dot{e}_{a}^{\mu} \dot{\omega}_{b \sigma}^{a} \dot{e}_{\rho}^{b}=\dot{e}_{a}{ }^{\mu} \dot{\partial}_{\sigma} \dot{e}_{\rho}^{a},
$$

which is the the Weitzenböck connection revealing the Weitzenböck spacetime $W_{4}$ of the teleparallel gravity. Thus, $\dot{\pi}(\dot{x})$ can be referred to as the Weitzenböck deformation matrix. The Weitzenböck connection is a connection presenting a nonvanishing torsion, but a vanishing curvature. This recovers a particular case of the teleparallel gravity theory with the dynamical torsion. All magnitudes related to the teleparallel gravity will be denoted with an over “.”. Equations (56) and (57) are simply different ways of expressing the property that the total - that is, acting on both indices-derivative of the tetrad vanishes identically. According to the TSSDmap, the next first deformation matrices $\sigma(x):=\left(\sigma_{b}{ }^{a}\right)(x)$ contribute to corresponding ingredient parts according to (A.5)-(A.8). In particular, the tetrad changes according to

$$
\begin{gathered}
e_{c}=\sigma_{c}{ }^{d} \dot{e}_{d}, \quad \vartheta^{c}=\sigma_{e}^{c}{ }_{e} \dot{\vartheta}^{e}, \\
e_{c} \otimes \vartheta^{c}=\bar{\chi}_{d}{ }^{c} \dot{e}_{c} \otimes \dot{\vartheta}^{d}=\Omega_{b}{ }^{a} \breve{e}_{a} \otimes \breve{\vartheta}^{b},
\end{gathered}
$$

and a general spin connection transforms according to

$$
\omega_{b \mu}^{\prime a}=\sigma_{c}^{a} \dot{\omega}_{d \mu}^{c} \sigma_{b}^{d}+\sigma_{c}^{a} \partial_{\mu} \sigma_{b}^{c}
$$

So,

$$
\stackrel{(\sigma)}{\omega}_{b \mu}:=\omega_{b \mu}^{\prime a}=\sigma_{c}^{a} \partial_{\mu} \sigma_{b}^{c}
$$

is referred to as a $G L(4, R)$ valued deformation-related frame connection, which represents the deformed properties of the frame only. Then, it follows that the affine connection, $\Gamma$, related to (28) and (58) tetrad deformations, transforms through

$$
\begin{aligned}
\Gamma_{\rho \sigma}^{\mu} & =e_{a}^{\mu} \partial_{\sigma} e_{\rho}^{a}+e_{a}^{\mu}{\stackrel{(\pi)}{\omega} a_{b \sigma} e_{\rho}^{b}} \\
& =\sigma_{a}^{\mu} \partial_{\sigma} \sigma_{\rho}^{a}+\sigma_{a}^{\mu}{\stackrel{(\sigma)}{\omega} a_{b \sigma} \sigma_{\rho}^{b}}_{\rho}
\end{aligned}
$$

where, according to (A.7), we have $\sigma_{a}{ }^{\mu} \sigma_{\mu}{ }^{b}=\delta_{a}^{b}$, also the procedure can be inverted $\sigma_{a}{ }^{\mu} \sigma_{\nu}{ }^{a}=\delta_{v}^{\mu}$, and

$$
\stackrel{(\pi)}{\omega}_{b \mu}:=\omega^{a}{ }_{b \mu}=\pi^{a}{ }_{c} \breve{\omega}_{d \mu}^{c} \pi^{d b}+\pi^{a}{ }_{c} \partial_{\mu} \pi^{c b}
$$

is a more general $G L(4, R)$ valued spin connection. For our convenience, hereafter the notation, $\{\stackrel{(A)}{e} \underset{a}{ }, \stackrel{(A)}{\vartheta} b\}(A=\pi, \sigma)$, will be used for general linear frames

$$
\begin{aligned}
\left\{\begin{array}{c}
(A) \\
e_{a}
\end{array} \stackrel{(A)}{\vartheta} b\right\} & =\left\{\left(\begin{array}{c}
(\pi) \\
e_{a}, \stackrel{(\sigma)}{e}_{a}
\end{array}\right),\left(\begin{array}{c}
(\pi) \\
\vartheta
\end{array}, \stackrel{(\sigma)}{\vartheta} b\right)\right\} \\
& \equiv\left\{\left(e_{a}, \dot{e}_{a}\right),\left(\vartheta^{b}, \dot{\vartheta}^{b}\right)\right\},
\end{aligned}
$$

where $\left.\stackrel{(A)}{e}_{a}\right\rfloor \stackrel{(A)}{\vartheta^{b}}=\delta_{a}^{b}$, or in components, $\stackrel{(A)}{e}_{a}^{\mu} \stackrel{(A)}{e}_{\mu}=\delta_{a}^{b}$, and

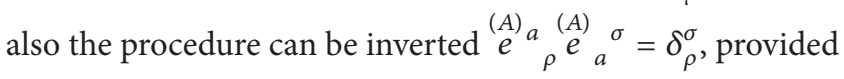

$$
\stackrel{(A)}{e} \underset{a}{\mu}=(\stackrel{(\pi)}{e} \underset{a}{\mu}, \stackrel{(\sigma)}{e} \underset{a}{\mu}) \equiv\left(e_{a}^{\mu}, \sigma_{a}^{\mu}\right) \text {. }
$$

The affine connection (61) can then be rewritten in the abbreviated form

$$
\Gamma_{\rho \sigma}^{\mu}=\stackrel{(A)}{e}_{a}^{\mu} \partial_{\sigma} \stackrel{(A)}{e}_{\rho}+\stackrel{(A)}{e}_{a}^{\mu} \stackrel{(A)}{\omega}_{b \sigma} \stackrel{(A)}{e}_{\rho} .
$$

Since the first deformation matrices $\pi(x)$ and $\sigma(x)$ are arbitrary functions, the transformed general spin connections $\stackrel{(\pi)}{\omega}(x)$ and $\stackrel{(\sigma)}{\omega}(x)$, as well as the affine connection (65), are independent of tetrad fields and their derivatives. In what follows, therefore, we will separate the notions of space and connections, the metric-affine formulation of gravity. A metric-affine space $\left(M_{4}, g, \Gamma\right)$ is defined to have a metric and a linear connection that need not be dependent on each other. The lifting of the constraints of metric-compatibility and symmetry yields the new geometrical property of the spacetime, which are the nonmetricity 1 -form $\stackrel{(A)}{N}_{a b}$ and the affine torsion 2 -form $\stackrel{(A)}{T}^{a}$ representing a translational misfit (for a comprehensive discussion see [35-37, 94]). These, together with the curvature 2 -form $\stackrel{(A)}{R}_{a}{ }^{b}$, symbolically can be presented as $[8,9]$

$$
\left(\stackrel{(A)}{N}{ }_{a b}, \stackrel{(A)}{T} a \stackrel{(A)}{R} \underset{a}{b}\right) \sim \stackrel{(A)}{\sim}\left(\stackrel{(A)}{g}_{a b}, \stackrel{(A)}{\mathcal{V}} a \stackrel{(A)}{\Gamma} \underset{a}{b}\right)
$$

where $\stackrel{(A)}{\mathscr{D}}$ is the covariant exterior derivative. It is well known that, due to the affine character of the connection space [115], one can always add a tensor to a given connection without spoiling the covariance of derivative (A.17). Let us define then a translation in the connection space. Suppose a point in this space will be a Lorentz connection, $\stackrel{(\pi)}{\omega}(x):=\stackrel{(\pi)}{\omega}_{b c}^{b}(x) J_{b c} d x^{\mu}$, presenting simultaneously curvature and torsion written in the language of differential forms as

$$
\begin{aligned}
& \stackrel{(\pi)}{R}=d \stackrel{(\pi)}{\omega}+\stackrel{(\pi)(\pi)}{\omega} \stackrel{(\omega)}{\underset{(\pi)}{\omega}} \underset{(\pi)}{\omega}, \\
& \stackrel{(\pi)}{T}=\operatorname{de}+\stackrel{(\pi)}{\omega} e \equiv \mathscr{D}_{(\pi)} e,
\end{aligned}
$$

where $\mathscr{D}_{(\pi)}$ denotes the covariant differential in the connection $\stackrel{(\pi)}{\omega}$. Now, given two connections $\stackrel{(\sigma)}{\omega}(x)$ and $\stackrel{(\pi)}{\omega}(x)$, 
the difference $k=\stackrel{(\sigma)}{\omega}-\stackrel{(\pi)}{\omega}$ is also a 1-form assuming values in the Lorentz Lie algebra, but transforming covariantly, whereas its covariant derivative is $\mathscr{D}_{(\pi)} k=d k+\{\underset{\omega}{\omega}, k\}$. The effect of adding a covector $k$ to a given connection $\stackrel{(\pi)}{\omega}$, therefore, is to change its curvature and torsion 2-forms:

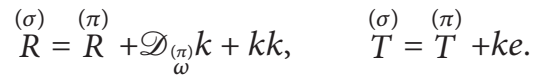

Since $k_{b c}^{a}$ is a Lorentz-valued covector, it is necessarily antisymmetric in the first two indices. Presenting $k_{b c}^{a}=$ $(1 / 2) k_{(b c)}^{a}+(1 / 2) k_{[b c]}^{a}$, we may define $k_{[b c]}^{a} \equiv t_{b c}^{a}$, such that

$$
k_{b c}^{a}=\frac{1}{2}\left(t_{a b}^{c}+t_{b a}^{c}-t_{b c}^{a}\right) .
$$

Turning to the connection appearing in the covariant derivative (A.17): $\stackrel{(\pi)}{\Omega}^{a}{ }_{b c} \equiv \stackrel{(\pi)}{\omega}^{a}{ }_{b c}-\stackrel{(\pi)}{K}^{a}{ }_{b c}$, a translation in the connection space with parameter $k_{b c}^{a}$ corresponds to

$$
\stackrel{(\sigma)}{\Omega}^{a}{ }_{b c}=\stackrel{(\pi)}{\Omega}^{a}{ }_{b c}+k_{b c}^{a}{ }_{b c} \stackrel{(\pi)}{\omega}^{a}{ }_{b c}-\stackrel{(\pi)}{K}^{a}{ }_{b c}+k_{b c}^{a} .
$$

Since $k_{b c}^{a}$ has always the form of a contortion tensor (69), the above connection is equivalent to $\stackrel{(\sigma)}{\Omega}^{a}{ }_{b c}=\stackrel{(\pi)}{\omega}^{a}{ }_{b c}-\stackrel{(\sigma)}{K}^{a}{ }_{b c}$, with $\stackrel{(\sigma)}{K}^{a}{ }_{b c}=\stackrel{(\pi)}{K}^{a}{ }_{b c}-k_{b c}^{a}$ being another contortion tensor: $\stackrel{(\sigma)}{K}_{\mu \nu}=\stackrel{(\pi)}{K}_{\mu \nu}-k_{\mu \nu}^{\lambda}$. There are actually infinitely many choices for $t_{b c}^{a}$, each one defining a different translation in the connection space and consequently yielding a connection $\stackrel{(\pi)}{\omega}{ }_{b c}$ with different curvature and torsion.

\section{Outline of the Key Points of TSSD Gauge MAG Theory}

At low energies the spacetime group associated with the matter fields is the Poincaré group. An extension of the Poincaré gauge theory of gravity constructed in the RC geometry, to the most general spacetime symmetry gauge theory, the MAG theory, has been developed by Hehl and collaborators $[17,18]$. The MAG theory has the most general type of covariant derivative: in addition to curvature and torsion, the MAG also has nonmetricity, that is, a nonmetric compatible connection. Hence parallel transport no longer preserves length and angle. Note that there are only indications (but no conclusive evidence) for assuming invariance of physical systems under the action of the entire affine group and that in MAG one is far from actually calculating $S$-matrix elements. Although the theoretical structure of this theory has been developed, we do not yet have much understanding of what new physics are allowed by the MAG theory. One source of improved understanding is exact solutions; see, for example, [116-118] and references therein. But due to the highly nonlinear nature of theory, exact solutions are not easily found unless they have a great deal of symmetry. However, to carry in full generality through the extension of
TSSD-ideas as applied to more general metric-affine gravity, it reasonable as a next step to gauge immediately the $4+16$ parameter affine group $A(4, R)=R^{4} \otimes G L(4, R)$, which lacks a metric structure altogether, and to introduce the metric subsequently. General affine invariance adds dilation and shear invariance as physical symmetries to Poincaré invariance, and both of these symmetries are of physical importance. Dilation invariance is a crucial component of particle physics in the high energy regime. Shear invariance was shown to yield representations of hadronic matter; the corresponding shear current can be related to hadronic quadrupole excitations. From this in the framework of the gauge theory of the affine group with a metric supplemented, as a physically meaningful field theory, it is speculated that the invariance under affine transformations played an essential part at an early stage of the universe, such that todays Poincaré invariance might be a remnant of affine invariance after some symmetry breaking mechanism. Thus MAG encompasses the PG as a subcase. The rather comprehensive gauging of the affine group was done in $[17,18]$, where a rigorous mathematical justification of the relevant spacetime structures and equations with more details is to be found. But a brief outline of the key points of relevance to the context of TSSD can be stated here, in which some conventions and results will be borrowed from the presentation given in $[17,18,42]$. To this aim, one enlarges at any point of the base manifold $x \in \mathscr{M}$ a tangent space $T_{x} \mathscr{M}_{4}$ to an affine tangent space $A_{x} \mathscr{M}_{4}$ by allowing freely translating elements of $T_{x} \mathscr{M}_{4}$ to different points $p \in A_{x} \mathscr{M}_{4}$. The collection of all affine tangent spaces $A_{x} \mathscr{M}_{4}$ forms the affine bundle $A \mathscr{M}_{4}$. An affine frame of $\mathscr{M}_{4}$ at $x$ is a pair $(\stackrel{(A)}{e} \underset{a}{a}, p)$ (whereas before $A=\pi, \sigma)$ consisting of a linear frame $\stackrel{(A)}{e}_{a} \in L_{x} \mathscr{M}_{4}$ and a point $p \in A_{x} \mathscr{M}_{4}$. The origin of $A_{x} \mathscr{M}_{4}$ is that point $o_{x} \in A_{x} \mathscr{M}$ for which the affine frame $\left(\stackrel{(A)}{e} \underset{a}{a}, o_{x}\right) \in A_{x} \mathscr{M}_{4}$ reduces to the linear frame $\stackrel{(A)}{e}_{a} \in L_{x} \mathscr{M}_{4}$. The transformation behavior of an affine frame $\left(\stackrel{(A)}{e}_{a}, p\right)$ under an affine transformation $(\Lambda, \tau)$ with $\tau=\tau^{a} \in T^{4} \simeq R^{4}$ and $\Lambda=\Lambda_{a}^{b} \in G L(4, R)$ reads

$$
\begin{aligned}
(\stackrel{(A)}{e}, p) \stackrel{(\Lambda, \tau)}{\longrightarrow}\left(\stackrel{(A)}{e}, p^{\prime}\right) & =(\stackrel{(A)}{e} \Lambda, p+\tau) \\
& =\left(\stackrel{(A)}{e}_{b} \Lambda_{a}^{b}, p+\tau^{a} \stackrel{(A)}{e}_{a}\right) .
\end{aligned}
$$

The affine group acts transitively on the affine tangent spaces $A \mathscr{M}_{4}$ : any two affine frames of some $A_{x} \mathscr{M}_{4}$ can be related by a unique affine transformation. Consequently, the notion of an affine frame should be enlarged to include all $G L(4, R)$ representations needed. The gauging is accomplished by the introduction of the generalized affine connection as a prescription $(\stackrel{(A)}{\Gamma}(L), \stackrel{(A)}{\Gamma}(T)$, which maps infinitesimally neighbouring affine tangent spaces $A_{x} \mathscr{M}_{4}, A_{\tilde{x}} \mathscr{M}_{4}$, where $\tilde{x}=x+d x$, by an $A(4, R)$-transformation onto each other. The generalized affine connection consists of a $G L(4, R)$-valued 1-form $\stackrel{(A)}{\Gamma}(L)$ and an $R^{4}$-valued one-form $\stackrel{(A)}{\Gamma}^{(T)}$, both of which generate the required $A(4, R)$-transformation. The two affine tangent 
spaces get now related by an affine transformation according to the prescription

$$
d p=\stackrel{(A)}{\Gamma}(T) a\left(\stackrel{(A)}{e}_{a}, \quad d \stackrel{(A)}{e}_{a}=\stackrel{(A)}{\Gamma} \underset{a}{(L) b} \stackrel{(A)}{e}_{b} .\right.
$$

Introducing origins in $A_{x} \mathscr{M}_{4}$, that is, by soldering $A_{x} \mathscr{M}_{4}$ to $\mathscr{M}_{4}$, one losts translational invariance in $A_{x} \mathscr{M}_{4}$ but gained a local one-to-one correspondence between translations in $A_{x} \mathscr{M}_{4}$ and diffeomorphisms on $\mathscr{M}_{4}$. However, one can introduce translational invariance by demanding diffeomorphism invariance instead and continue to work with this modified notion of translation invariance. The diffeomorphisms themself, as horizontal transformations in their active interpretation, cannot be gauged according to the usual gauge principle and thus do not furnish their own gauge potential. In the Lagrangian formulation of a general metric-affine theory it was assumed that the matter fields are described in terms of manifields. Actually, the matter fields are supposed to be represented by vector- or spinorvalued $p$-forms. However, in MAG one goes beyond Poincaré invariance, assuming that matter fields might undergo not only Poincaré transformations but also the more general linear transformations. In this case, the unavailability of local Lorentz frames poses no problem in the context of boson fields. They are naturally constructed so as to be capable of carrying the action of $S L(4, R)$, instead of the Lorentz group, whether in a local frame or holonomically. However, this is not true of the conventional fermion fields one uses to represent matter, and a linear action should nevertheless be realized, through the use of infinite-component linear field representations of the double covering of the linear, affine, and diffeomorphism groups. More general spinor representations than the Poincaré-representations have to be constructed. Such representations of the matter fields corresponding to the affine group must exist. Otherwise it does not make sense to demand $A(4, R)$-invariance of a nonvacuum field theory. The construction of the spinor representations of fermionic matter fields in MAG, the so-called manifields, is illustrated in $[17,18]$. These representations turn out to be infinite dimensional, due to the noncompactness of the gauge (sub)group $G L(4, R)$. Fermions are then assigned to spinor manifields. The restriction of $G L(4, R)$ to $S O(1, n-1)$ reduces the manifield representations to the familiar spinor representations. The actual gauging of the affine group introduced, in addition to the matter fields $f$, the gravitational gauge potentials $\stackrel{(A)}{\Gamma}^{(T)}$ and $\stackrel{(A)}{\Gamma}^{(L)}$. Following the common practice, we will use as gauge potential the translation invariant $\stackrel{(A)}{\vartheta} a$ in place of the translational part $\stackrel{(A)}{\Gamma}^{(T)}$ of the affine connection, simply because it has the immediate interpretation as a reference (co)frame. Expanded in a holonomic frame, the components of $\stackrel{(A)}{\vartheta} a$ and $\stackrel{(A)}{\Gamma}(T)$ differ just by a Kronecker symbol, as is clear from the definition of $\underset{\vartheta}{\stackrel{(A)}{a}}$. Also the homogeneous transformation behavior of $\stackrel{(A)}{\vartheta} a$ will turn out to be quite convenient. For the action of the $G L(4, R)$-gauge potential, below the shorthand notation $\stackrel{(A)}{\Gamma}{ }_{a}{ }^{b}$ is used instead of $\left(\stackrel{(A)}{\Gamma}{ }_{c}^{(L) d} L_{d}^{c}\right)_{a}^{b}$. The introduction of a metric into MAG is mandatory since we are interested in a realistic macroscopic gravity theory that contains GR in some limit. So, it was assumed as a metric of the general form $\stackrel{(A)}{g}=\stackrel{(A)}{g} a b \stackrel{(A)}{9} a \otimes \stackrel{(A)}{\vartheta} b$ with coefficients $\stackrel{(A)}{g}_{a b}$ which are independent of the coframe $\stackrel{(A)}{\vartheta}^{a}$. The gauge potentials $\left(\stackrel{(A)}{\vartheta^{a}}, \stackrel{(A)}{\Gamma}{ }_{a} b\right)$ become true dynamical variables if one has to add to the minimally coupled matter Lagrangian $L_{m}^{(\pi)}$ a gauge Lagrangian $V$. Here, we restrict ourself considering only first order Lagrangian $V$, which must be expressed in terms of the gauge potentials and their first derivatives. The total action reads then

$$
\begin{aligned}
& S=\int\left[V\left(\stackrel{(A)}{g}_{a b}, \stackrel{(A)}{\vartheta} a, \stackrel{(A)}{N}_{a b}, \stackrel{(A)}{T} a, \stackrel{(A)}{R}_{a}^{b}\right)\right. \\
& \left.+L_{m}^{(\pi)}\left(\stackrel{(A)}{g}_{a b}, \stackrel{(A)}{\vartheta}^{a}, \Psi, D \Psi\right)\right] .
\end{aligned}
$$

If $\Psi$, as a $p$-form, represents a matter field (fundamentally a representation of the $S L(4, R)$ or of some of its subgroups), its first order Lagrangian $L_{m}^{(\pi)}$ will be embedded in metricaffine spacetime by the minimal coupling procedure, that is, exterior covariant derivatives feature in the kinetic terms of the Lagrangian instead of only exterior ones. Just as ordinary stress is the analogue of the (Hilbert) energy-momentum density, in MAG, one has, in addition, the spin current and the dilation plus shear currents inducing the torsion and nonmetricity fields, respectively. Both spin and dilation plus shear are components of the hypermomentum current, symmetric for dilation plus shear and antisymmetric for spin: spincurrent $\otimes$ dilation current $\otimes$ shear current. And these currents ought to couple to the corresponding postRiemannian structures. In accord, the material currents are defined as follows:

$$
\begin{gathered}
\stackrel{(A)}{t} a b^{a}:=2 \frac{\delta L_{m}^{(\pi)}}{\delta \stackrel{(A)}{g}_{a b}}, \quad \stackrel{(A)}{\theta}_{a}:=\frac{\delta L_{m}^{(\pi)}}{\delta \stackrel{(A)}{\vartheta}^{a}}, \\
{\stackrel{(\pi)}{\Delta}{ }_{b}}_{b}:=\frac{\delta L_{m}^{(\pi)}}{\delta \stackrel{(\pi)}{\Gamma}_{a}^{b}},
\end{gathered}
$$

where ${ }^{(A)} a b$ is the metric (and symmetric) energy-momentum current of matter (Hilbert current), whereas one believes $(A)$

that $\stackrel{(A)}{\theta}_{a}$ should be the dual 3 -form relating to the canonical energy-momentum tensor, $\stackrel{(A)}{\theta} \underset{a}{\mu}$, by

$$
\star \stackrel{(A)}{\theta}_{a}=\frac{1}{3 !} \stackrel{(A)}{\theta}_{a}^{\mu} \varepsilon_{\mu \nu \alpha \beta} \stackrel{(A)}{\vartheta} \nu \alpha \beta,
$$

where we used the abbreviated notations for the wedge product monomials, $\stackrel{(A)}{\vartheta} \mu \nu \alpha \cdots=\stackrel{(A)}{\vartheta} \mu \wedge \stackrel{(A)}{\vartheta}{ }^{\vartheta} \wedge \stackrel{(A)}{\vartheta} \alpha \wedge \cdots$, and $\star$ denotes the Hodge dual (Appendix B). The canonical energy-momentum density is identical with the dynamical tetrad energy-momentum density $e_{\theta_{\mu}}^{(A)} a=\stackrel{(A)}{\tau}_{\mu} a$, where 
$e:=\operatorname{det}\left|e_{a}^{\mu}\right|=\sqrt{-g}$, and the canonical tensor $e^{(A)} \theta_{\mu \nu}=\stackrel{(A)}{\tau}_{\mu \nu}=$ $\stackrel{(A)}{e}_{a v}{ }^{(A)}{ }_{\mu} a$ is generally not symmetric. The relation between the tetrad dynamical energy-momentum tensor and the metric dynamical energy-momentum tensor for matter fields is $\stackrel{(A)}{\theta}_{(\mu \nu)}=t_{\mu \nu}$. The canonical hypermomentum current $\stackrel{(\pi)}{\Delta}{ }^{a}{ }_{b}$, which couples to the linear connection, can be decomposed according to

$$
\stackrel{(\pi)}{\Delta}_{a b}=\stackrel{(\pi)}{S}_{a b}+\frac{1}{4} \stackrel{(\pi)}{g}_{a b} \Delta+\widehat{\Delta}_{\alpha \beta}
$$

with $\stackrel{(\pi)}{S}_{a b}:=\stackrel{(\pi)}{\Delta}_{[a b]}$ as (dynamical) spin current, $\Delta:=\stackrel{(\pi)}{\Delta}_{c}^{c}$, as dilation current, and $\widehat{\Delta}_{a b}$ as symmetric and tracefree shear current, which is a bit more remote from direct observation than the other currents. From variation of a total action (73), we find the matter and the gauge field equations as follows:

$$
\begin{aligned}
& D\left(\frac{\partial L_{m}^{(\pi)}}{\partial(D \Psi)}\right)-(-1)^{p} \frac{\partial L_{m}^{(\pi)}}{\partial \Psi}=0, \quad \text { (matter) } \\
& D\left(2 \frac{\partial V}{\partial N_{a b}^{(A)}}\right)+2 \frac{\partial V}{\partial \stackrel{(A)}{g}_{a b}}=-\stackrel{(A)}{t} a b \\
& D\left(\frac{\partial V}{\partial \stackrel{(A)}{T}^{a}}\right)+\frac{\partial V}{\partial \stackrel{(A)}{\vartheta}}=-\stackrel{(A)}{\theta}_{a}, \\
& \frac{\partial \stackrel{(A)}{\omega} \underset{a^{\prime}}{b^{\prime}}}{\partial \stackrel{(\pi)}{\omega}_{a}^{b}} \wedge\left[D\left(\frac{\partial V}{\partial \stackrel{(A)}{R}{ }_{a^{\prime}} b^{\prime}}\right)+\stackrel{(A)}{\vartheta} a^{\prime} \wedge \frac{\partial V}{\partial \stackrel{(A)}{T} b^{\prime}}\right. \\
& \left.+2 \stackrel{(A)}{g}_{b^{\prime} c} \frac{\partial V}{\partial \stackrel{(A)}{N}_{a^{\prime} c}}\right]=-\stackrel{(\pi)}{\Delta}^{a}{ }_{b},
\end{aligned}
$$

where we take into account that the variation of $L_{m}^{(\pi)}$ with respect to the affine connection in MAG is equivalent to the variation with respect to the torsion (or contortion) or spin

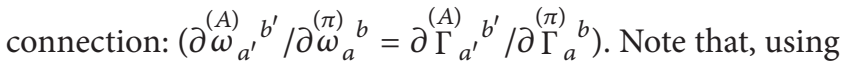
the Noether identities, the zeroth field equation can be shown to be redundant, provided the matter equations hold. In first, the canonical energy-momentum of the translational gauge potential $\stackrel{(A)}{\vartheta} a$ can be written in standard form

$$
\begin{aligned}
& \left.\left.\frac{\partial V}{\partial \stackrel{(A)}{\vartheta^{a}}}=\stackrel{(A)}{e}_{a}\right\rfloor V-\left(\stackrel{(A)}{e_{a}}\right\rfloor \stackrel{(A)}{T} b\right) \wedge \frac{\partial V}{\partial \stackrel{(A)}{T} b} \\
& \left.\left.-\left(\stackrel{(A)}{e}_{a}\right\rfloor \stackrel{(A)}{R}_{b}^{c}\right) \wedge \frac{\partial V}{\partial \stackrel{(A)}{R}_{b} c}-\left(\stackrel{(A)}{e}_{a}\right\rfloor \stackrel{(A)}{N}_{b c}\right) \frac{\partial V}{\partial \stackrel{(A)}{N}_{b c}} .
\end{aligned}
$$

Thus, the equations of the standard MAG theory written in the framework of the first order Lagrangian, which is expressed in terms of the gauge potentials and their first derivatives, can be recovered for $A=\pi$ :

$$
\begin{aligned}
& D\left(\frac{\partial L_{m}^{(\pi)}}{\partial(D \Psi)}\right)-(-1)^{p} \frac{\partial L_{m}^{(\pi)}}{\partial \Psi}=0, \quad \text { (matter) } \\
& D\left(2 \frac{\partial V}{\partial \stackrel{(\pi)}{N}_{a b}}\right)+2 \frac{\partial V}{\partial \stackrel{(\pi)}{g}_{a b}}=-\stackrel{(\pi)}{t}^{a b}, \\
& D\left(\frac{\partial V}{\partial T^{a} a}\right)+\frac{\partial V}{\partial \stackrel{(\pi)^{a}}{\vartheta}}=-\stackrel{(\pi)}{\theta}_{a}, \\
& D\left(\frac{\partial V}{\partial \stackrel{(\pi)}{R}_{a^{\prime}} b^{\prime}}\right)+\stackrel{(\pi)}{\vartheta} a^{\prime} \wedge \frac{\partial V}{\partial \stackrel{(\pi)}{T}^{b^{\prime}}}+2 \stackrel{(\pi)}{g}_{b^{\prime} c} \frac{\partial V}{\partial \stackrel{(\pi)}{N}_{a^{\prime} c}} \\
& =-\stackrel{(\pi)}{\Delta}^{a}{ }_{b}, \quad \text { (2nd). }
\end{aligned}
$$

Consequently, the (2nd) equation defines a nondynamical torsion, such that torsion at a given point in spacetime does not vanish only if there is matter at this point, represented in the Lagrangian density by a function which depends on torsion. Unlike the metric, which is related to matter through a differential field equation, torsion does not propagate. However, equations (79) can be equivalently replaced by the set of modified MAG equations for $A=\sigma$ :

$$
\begin{aligned}
& D\left(\frac{\partial L_{m}^{(\pi)}}{\partial(D \Psi)}\right)-(-1)^{p} \frac{\partial L_{m}^{(\pi)}}{\partial \Psi}=0, \quad \text { (matter) } \\
& D\left(2 \frac{\partial V}{\partial \stackrel{(\sigma)}{N}_{a b}}\right)+2 \frac{\partial V}{\partial \stackrel{(\sigma)}{g}_{a b}}=-\stackrel{(\sigma)}{t}^{a b}, \\
& D\left(\frac{\partial V}{\partial \stackrel{(\sigma)}{T} a}\right)+\frac{\partial V}{\partial \stackrel{(\sigma)^{a}}{\vartheta}}=-\stackrel{(\sigma)}{\theta}_{a} \\
& \Theta_{a^{\prime} b}^{a b^{\prime}}(\pi(x), \sigma(x)) \wedge\left[D\left(\frac{\partial V}{\partial \stackrel{(\sigma)}{R} a_{a^{\prime}} b^{\prime}}\right)+\stackrel{(\sigma)}{\vartheta} a^{\prime} \wedge \frac{\partial V}{\partial \stackrel{(\sigma)}{T} b^{\prime}}\right. \\
& \left.+2 \stackrel{(\sigma)}{g}_{b^{\prime} c} \frac{\partial V}{\partial N_{a^{\prime} c}^{(\sigma)}}\right]=-\stackrel{(\pi)}{\Delta} a
\end{aligned}
$$

where

$$
\frac{\partial \stackrel{(\sigma)}{\omega} a_{a^{\prime}}^{b^{\prime}}}{\partial \stackrel{(\pi)}{\omega}_{a}^{b}}:=\Theta_{a^{\prime} b}^{a b^{\prime}}(\pi(x), \sigma(x)),
$$

in which the torsion is dynamical if only $\Theta_{a^{\prime} b}^{a b^{\prime}}(\pi(x), \sigma(x)) \neq$ $\delta_{a}^{a^{\prime}} \delta_{b^{\prime}}^{b}$. Actually, in testing the general MAG equations (77), we desire, in some limit, to recover the field equation 
for different (sub)cases; then we have to put on Lagrange multipliers, whereas, in the case of TSSD-PG, one has to kill nonmetricity; TSSD-EC is the TSSD-PG with the curvature scalar as gravitational Lagrangian; in the case of TSSDteleparallel gravity (TSSD-GR $\mathrm{R}_{||}$) in a Weitzenböck spacetime, one has to remove nonmetricity and curvature; and, finally, in the case of GR in a Riemannian space, curvature scalar as Lagrangian, one has to remove nonmetricity and torsion. To recover, for example, GR, let us in (68) choose $t^{a}{ }_{b c}$ as the torsion of the connection $\stackrel{(\pi)}{\omega}^{a}{ }_{b c}$; that is, $t_{b c}^{a}=\stackrel{(\pi)}{T}^{a}{ }_{b c}$. In this case, $k_{b c}^{a}=\stackrel{(\pi)}{K}^{a}{ }_{b c}$, and we are left with the torsionless, a $S O(3,1)$ valued Lorentz spin connection of general relativity: $\stackrel{(\sigma)}{\Omega}^{a}{ }_{b c}=\breve{\omega}^{a}{ }_{b c}$. Then, one can choose for the missing piece of the gauge field Lagrangian $V_{\mathrm{GR}}$ the corresponding 4 -form of the curvature scalar $[8,9]$ and take Lagrange multipliers for extinguishing nonmetricity and torsion:

$$
V_{\mathrm{GR}}=-\frac{1}{4 æ} \breve{R}^{a b} \wedge \breve{\eta}_{a b}+\frac{1}{2} \stackrel{(A)}{N}_{a b} \wedge{ }^{(1)} \lambda^{a b}+\stackrel{(A)}{T}^{a} \wedge^{(2)} \lambda_{a}
$$

where $æ$ is the coupling constant relating to the Newton gravitational constant $æ=8 \pi G / c^{4}, \breve{R}_{a}^{b}$ is the curvature tensor of the independent field variable $\breve{\Gamma}_{a}^{b}$, and $\breve{\eta}_{a b}$ is the eta basis consisting in the Hodge dual of exterior products of tetrads by means of the Levi-Civita object (Appendix B).

The RC manifold, $U_{4}$, is a particular case of general metric-affine manifold $M_{4}$, restricted by the metricity condition, when a nonsymmetric linear connection is said to be metric compatible. Taking the antisymmetrized derivative of the metric condition gives an identity between the curvature of the spin connection and the curvature of the Christoffel connection

$$
\stackrel{(A)}{R}_{\mu \nu}^{a b}(\stackrel{(A)}{\omega}) \stackrel{(A)}{e}_{\rho b}-R_{\rho \mu \nu}^{\sigma}(\Gamma) \stackrel{(A)}{e}_{\sigma}^{a}=0
$$

where we take into account (65), so

$$
\begin{aligned}
& \stackrel{(A)}{R}_{\mu \nu}^{a b}(\stackrel{(A)}{\omega})=\partial_{\mu} \stackrel{(A)}{\omega}_{\nu}^{a b}-\partial_{\nu} \stackrel{(A)}{\omega}_{\mu}^{a b} \\
& +\stackrel{(A)}{\omega}_{\mu} a c \stackrel{(A)}{\omega}_{\nu c}^{b}-\stackrel{(A)}{\omega}_{\nu} a c \stackrel{(A)}{\omega}_{\mu c}^{b}, \\
& R_{\rho \mu \nu}^{\sigma}(\Gamma)=\partial_{\mu} \Gamma_{\nu \rho}^{\sigma}-\partial_{\nu} \Gamma_{\mu \rho}^{\sigma}-\Gamma_{\mu \rho}^{\lambda} \Gamma_{\nu \lambda}^{\sigma}+\Gamma_{\nu \rho}^{\lambda} \Gamma_{\mu \lambda}^{\sigma} .
\end{aligned}
$$

Hence, the relations between the scalar curvatures for the $U_{4}$ manifold read

$$
\begin{aligned}
\stackrel{(A)}{R}(\stackrel{(A)}{\omega}) & \equiv \stackrel{(A)}{e}_{a}^{\mu} \stackrel{(A)}{e}_{b} \stackrel{(A)}{R}_{\mu \nu}^{a b}(\stackrel{(A)}{\omega}) \\
& =R(g, \Gamma) \equiv g^{\rho v} R_{\rho \mu \nu}^{\mu}(\Gamma) .
\end{aligned}
$$

This means that the Lorentz and diffeomorphism invariant scalar curvature, $R$, becomes either a function of $\stackrel{(A)}{e}{ }_{\mu}^{a}$ only or a function of $g_{\mu \nu}$ only. Certainly, it can be seen by noting that the Lorentz gauge transformations can be used to fix the six antisymmetric components of $\stackrel{(A)}{e} \underset{\mu}{a}$ to vanish. Then in both cases diffeomorphism invariance fixes four more components out of the six $g_{\mu \nu}$, with the four components $g_{0 \mu}$ being nondynamical, obviously, leaving only two dynamical degrees of freedom. This shows the equivalence of the vierbein and metric formulations holds. To recover the TSSD$U_{4}$ theory, one can choose for the missing piece the EC Lagrangian $V_{\mathrm{EC}}$ (here without cosmological constant):

$$
V_{\mathrm{EC}}=-\frac{1}{4 æ} \stackrel{(A)}{R}^{a b} \wedge \stackrel{(A)}{\eta}_{a b}+\frac{1}{2} \stackrel{(A)}{N}_{a b} \wedge \lambda^{a b}
$$

provided $(1 / 2) \stackrel{(A)}{R} a b \wedge \stackrel{(A)}{\eta}$ ab is equal to $\stackrel{(A)(A)}{R} \stackrel{(A)}{\eta}^{\text {. The variation }}$ of the total action (73), given by the sum of the gravitational field action $S_{\mathrm{EC}}=\int V_{\mathrm{EC}}$ with the Lagrangian (86) and the macroscopic matter sources $S_{m}^{(\pi)}$, with respect to the $\stackrel{(A)}{\vartheta} a, 1-$ form $\stackrel{(A)}{\omega}^{a b}=\stackrel{(A)}{\omega}_{\mu}^{a b} \wedge d x^{\mu}$ and $\Psi$, gives

$$
\begin{aligned}
& \text { (1) } \frac{1}{2} \stackrel{(A)}{\eta}_{a b c} \wedge \stackrel{(A)}{R} b c=-æ \stackrel{(A)}{\theta}_{a}, \\
& \text { (2) } \Theta_{a b}^{a^{\prime} b^{\prime}} \wedge D \stackrel{(A)}{\eta}_{a b}=æ \stackrel{(\pi)}{S}_{a b}, \\
& \text { (3) } \frac{\delta L_{m}^{(\pi)}}{\delta \Psi}=0, \quad \frac{\delta L_{m}^{(\pi)}}{\delta \bar{\Psi}}=0,
\end{aligned}
$$

where $\partial \stackrel{(A)}{\omega} a^{\prime} b^{\prime} / \partial \stackrel{(\pi)}{\omega} a b:=\Theta_{a^{\prime} b^{\prime}}^{a b}(\pi(x), \sigma(x))$. The metricity condition $\stackrel{(A)}{N}_{a b}=\stackrel{(A)}{\mathscr{D}} \mathscr{g}_{a b}=0$ yields $\stackrel{(\pi)}{S}_{a b}+\stackrel{(\pi)}{S}_{b a}=0$, and hence $D \stackrel{(A)}{\eta}_{a b}=\stackrel{(A)}{\eta}_{a b c} \wedge \stackrel{(A)}{T}^{c}$. Consequently, the field equations (87) become

$$
\begin{aligned}
& \text { (1) } \frac{1}{2} \breve{R}_{c a}^{(A)} \wedge \stackrel{(A)}{\vartheta}^{c}=æ \stackrel{(A)}{\theta} a, \\
& \text { (2) } \Theta_{a b}^{a^{\prime} b^{\prime}} \wedge \star \stackrel{(A)}{\mathscr{T}}_{a^{\prime} b^{\prime}}=-\frac{1}{2} æ \star \stackrel{(\pi)}{\Sigma}_{a b}, \\
& \text { (3) } \frac{\delta L_{m}^{(\pi)}}{\delta \Psi}=0, \quad \frac{\delta L_{m}^{(\pi)}}{\delta \bar{\Psi}}=0,
\end{aligned}
$$

where $\star \Sigma_{a b}^{(\pi)}=-\star \sum_{b a}$ is the dual 3-form corresponding to the canonical spin tensor, which is identical with the dynamical spin tensor $\stackrel{(\pi)}{S}_{a b c}$; namely,

$$
\star \sum_{a b}^{(\pi)}=\stackrel{(\pi)}{S}_{a b}^{\mu} \varepsilon_{\mu \nu \alpha \beta} \stackrel{(\pi)}{\vartheta} \nu \alpha \beta
$$

provided

$$
\begin{aligned}
\star \stackrel{(A)}{\mathscr{T}}_{a b} & :=\frac{1}{2} \star\left(\stackrel{(A)}{Q}_{a} \wedge \stackrel{(A)}{e}_{b}\right)=\stackrel{(A)}{Q}^{c} \wedge \stackrel{(A)}{\wedge} d^{\varepsilon_{c d a b}} \\
& =\frac{1}{2} \stackrel{(A)}{Q}^{c}{ }_{\mu \nu} \wedge \stackrel{(A)}{e}_{\alpha} \varepsilon_{a b c d} \stackrel{(A)}{\vartheta}^{\mu \nu \alpha},
\end{aligned}
$$

and that

$$
\stackrel{(A)}{Q}^{a}=\stackrel{(A)(A)}{D} \stackrel{9}{\vartheta}=d \stackrel{(A)}{\vartheta}^{a}+\stackrel{(A)}{\omega}_{a}{ }_{b} \wedge \stackrel{(A)}{\vartheta} b
$$


According to (85), the relations between the Ricci scalars read

$$
\breve{R} \equiv \breve{R}_{c d}^{(\sigma)} \wedge \dot{\vartheta}^{c} \wedge \dot{\vartheta}^{d}=\breve{R}_{c d}^{(\pi)} \wedge \vartheta^{c} \wedge \vartheta^{d}
$$

To obtain some feeling for the tensor language then we may recast the first equation in (88) into the form

$$
-\frac{e}{4} \breve{R}_{\mu \nu}^{(A) a b} \stackrel{(A)}{e}_{a b c}=æ \stackrel{(A)}{\theta}_{c}^{a} e e_{e}^{(A)}{ }_{a}^{\beta} .
$$

Making use of the relation,

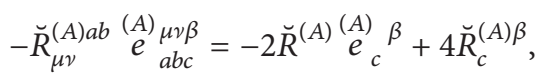

gives

$$
\breve{G}_{c}^{(A) \beta} \equiv \breve{R}_{c}^{(A) \beta}-\frac{1}{2} \stackrel{(A)}{e}_{c}^{\beta} \breve{R}=æ \stackrel{(A)}{\theta}_{\alpha}
$$

We may evaluate the second equation in (88) as

$$
\begin{aligned}
& \frac{\partial \stackrel{(A)}{\omega}_{\beta}^{c d}}{\partial{\stackrel{(\pi)}{\omega}{ }_{\beta^{\prime} d^{\prime}}}_{e}} \frac{(A)}{2}{ }^{a}{ }_{\mu \nu} \stackrel{(A)}{e}_{a v \beta}^{\mu \nu d}=-\frac{e}{2} æ \stackrel{(\pi)}{S}_{a}^{a} c_{c^{\prime} d^{\prime}} \stackrel{(A)}{e}_{a}^{\beta^{\prime}} \\
& =-\frac{e}{2} æ \stackrel{(\pi)}{S}_{\beta^{\prime}}{ }_{c^{\prime} d^{\prime}} .
\end{aligned}
$$

Taking into account the relation

$$
\frac{1}{2} \stackrel{(A)}{Q}^{c}{ }_{\mu \nu} \stackrel{(A)}{e}_{c a b}^{\mu \nu \beta}=\stackrel{(A)}{e}_{a}^{\mu} \stackrel{(A)}{e}_{b}^{v} \stackrel{(A)}{T}_{\mu \nu}^{\beta}
$$

we then obtain

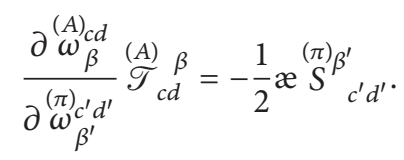

It is remarkable that the torsion (98), in a natural way, can be made a short-range propagating torsion. Actually, from the above equations, we see that it is the spin $\stackrel{(\pi)}{S}$ and spacetime deformations $\pi(x)$ and $\sigma(x)$ that define the torsion $\stackrel{(A)}{Q}$ :

$$
\stackrel{(A)}{Q}_{\mu \rho}=\stackrel{(A)}{T}_{\mu \rho}+\frac{1}{2} \delta_{\mu}^{\nu} \stackrel{(A)}{T}_{\rho \lambda}-\frac{1}{2} \delta_{\rho}^{\nu} \stackrel{(A)}{T}_{\mu \lambda}^{\lambda},
$$

which through the field equation, in turn, defines Einstein's field tensor $\breve{G}$. A generic spacetime deformation, $\pi(x)$, consists of two ingredient deformations $(\dot{\pi}(\dot{x}), \sigma(x))$ of the orthonormal frame. While the deformation matrix $\dot{\pi}(\dot{x})$ implies a peculiar condition (53), the choice of the $\sigma(x)$ is not fixed yet. This allows us to impose a physical constraint upon the spacetime deformation $\sigma(x)$ :

$$
\Theta_{\nu^{\prime} \mu \rho}^{\mu^{\prime} \rho^{\prime} v}(\pi(x), \sigma(x))=\left(\square+M_{T}^{2}\right) \stackrel{(\sigma)}{T}_{\mu \rho}^{\nu}\left(\stackrel{(\sigma)}{T}^{-1}\right)_{\nu^{\prime}}^{\mu^{\prime} \rho^{\prime}},
$$

where $\square$ is a generalization of the d'Alembertian operator for covariant derivatives defined on the RC manifold, $U_{4}$. Then, the modified EC equations (95) and (98) reduce to

$$
\breve{G}_{\mu \nu}=æ \stackrel{(\sigma)}{\theta}_{\mu \nu}, \quad\left(\square+M_{T}^{2}\right) \stackrel{(\sigma)}{T}_{\mu \rho}=-\frac{1}{2} æ \stackrel{(\pi)}{S}_{\mu \rho}^{\nu},
$$

which describe the short-range propagating torsion and spinspin interaction. At large distances $r>\lambda_{T} \equiv \hbar / M_{T} c$ (Compton length), torsion vanishes $\stackrel{(\sigma)}{T}(r)=0$. Note that the short-range propagating torsion is of fundamental importance from a view point of microphysics.

We may impose some other physical constraints upon the spacetime deformation $\sigma(x)$, which will be useful for the theory of electromagnetism and charged particles:

$$
\Theta_{\nu^{\prime} \mu \rho}^{\mu^{\prime} \rho^{\prime} \nu}(\pi(x), \sigma(x)) \equiv \Theta_{\nu^{\prime} \mu \rho}^{\mu^{\prime} \rho^{\prime} \nu}(\stackrel{(\sigma)}{T})=2 \varphi_{, \sigma} \varepsilon_{\mu \rho}^{\sigma \nu}(\stackrel{(\sigma)}{T}-1)_{\nu^{\prime}}^{\mu^{\prime} \rho^{\prime}}
$$

with $\varphi$ as a scalar or pseudoscalar function of relevant variables. Then

$$
\stackrel{(\pi)}{T}_{\mu \rho}^{\nu}=\Theta_{\nu^{\prime} \mu \rho}^{\mu^{\prime} \rho^{\prime} v}(\pi(x), \sigma(x)) \stackrel{(\sigma)}{T} \nu_{\mu^{\prime} \rho^{\prime}}^{\nu^{\prime}}=2 \varphi_{, \sigma} \varepsilon_{\mu \rho}^{\sigma \nu},
$$

which recovers the term in the Lagrangian of pseudoscalarphoton interaction theory [95-100], such that the nonmetric part of the Lagrangian can be put in the well known form of the $\chi-g$ framework:

$$
\begin{aligned}
L_{I}^{(\pi) N M} & =2(-g)^{1 / 2} A_{\nu} A_{\mu, \rho} \stackrel{(\pi)}{\mathscr{T}} \nu \mu \rho \\
& =4(-g)^{1 / 2} \varphi_{, \sigma} \varepsilon^{\sigma \nu \mu \rho} A_{\nu} A_{\mu, \rho}, \quad(\bmod \operatorname{div})
\end{aligned}
$$

where $F_{\mu \nu}=A_{\mu, \nu}-A_{\nu, \mu}$ have the usual meaning for electromagnetism. This is equivalent, up to integration by parts in the action integral (modulo a divergence), to the Lagrangian

$$
L_{I}^{(\pi) N M}=(-g)^{1 / 2} \varphi \varepsilon^{\sigma \nu \mu \rho} F_{\sigma \nu} F_{\mu \rho}
$$

According to (105), the gravitational constitutive tensor $\chi^{\sigma \nu \mu \rho}=\chi^{\mu \rho \sigma \nu}=-\chi^{\mu \rho \nu \sigma}$ [119] of the gravitational fields (e.g., metric $g_{\mu \nu}$, (pseudo) scalar field $\varphi$, etc.) reads

$$
\chi^{\sigma \nu \mu \rho}=(-g)^{1 / 2}\left[\frac{1}{2} g^{\sigma \mu} g^{\nu \rho}-\frac{1}{2} g^{\sigma \rho} g^{\mu \nu}+\varphi \varepsilon^{\sigma \nu \mu \rho}\right] .
$$

Specifying the physical meaning of the constrains (99), (101), and (102), will be an interesting topic for another investigation elsewhere. Here we finally concentrate on the other (sub)case when $t^{a}{ }_{b c}=\stackrel{(\pi)}{T}^{a}{ }_{b c}-\stackrel{(\pi)}{C}^{a}{ }_{b c}$, and the connection $\stackrel{(\pi)}{\omega}{ }_{b c}$ vanishes, which characterizes teleparallel gravity. In this case,

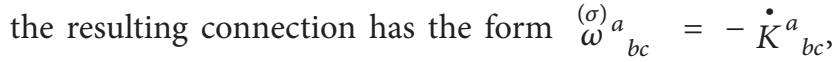
where $\dot{K}^{a}{ }_{b c}$ is the contortion tensor written in terms of the Weitzenböck torsion $\dot{T}^{a}{ }_{b c}=-\dot{C}^{a}{ }_{b c}$. The particle equation of motion then becomes the force equation of teleparallel 
gravity. Hence, the Weitzenböck covariant derivative of the tetrad field vanishes identically: $\dot{\mathscr{D}}_{\nu} \dot{e}_{\mu}{ }_{\mu} \equiv \dot{\partial}_{\nu} \dot{e}_{\mu}^{a}-$ $\dot{\Gamma}_{\mu \nu}^{\rho} \dot{e}_{\rho}^{a}=0$. This is the so-called distant, or absolute parallelism condition. As a consequence of this condition, the corresponding Weitzenböck spin connection also vanishes identically: $\dot{\omega}_{a v}^{c}=\breve{\omega}_{a v}^{c}+\dot{K}_{a v}^{c} \equiv 0$. Of course, these relations above are true only in one specific class of frames. In fact, since $\dot{\omega}^{c}$ av is the Weitzenböck spin connection, if it vanishes in a given frame, it will be different from zero in any other frame related to the first by a local Lorentz transformation. The teleparallel gravity becomes consistent and fully equivalent with GR, even in the presence of spinor fields if we write the minimal coupling prescription as $\dot{\partial}_{a} \rightarrow$ $\dot{\mathscr{D}}_{a}=\dot{e}{ }_{a}{ }^{\mu} \dot{\mathscr{D}}_{\mu}$ with $\dot{\mathscr{D}}_{\mu}$ the teleparallel Fock-Ivanenko derivative written in the form $\dot{\mathscr{D}}_{\mu}:=\dot{\partial}_{\mu}-(i / 2) \dot{\Omega}^{a}{ }_{b \mu} J_{a}{ }^{b}$, where the teleparallel spin connection, $\dot{\Omega}_{b \mu}^{a}$, reads $\dot{\Omega}_{b \mu}^{a}$ := $0-\dot{K}_{b \mu}^{a}$. Field equations can be derived from the least action, $\delta \dot{S}=0$, with the Lagrangian of teleparallel gravity, where we take Lagrange multipliers for extinguishing nonmetricity and curvature. This holds when the deformation $\sigma(x)$ vanishes identically $(\sigma(x) \equiv 0)$ :

$$
\begin{aligned}
V_{\|}= & -\frac{1}{4 æ} \dot{T}^{a} \wedge^{*}\left(-{ }^{(1)} \dot{T}_{a}+2^{(2)} \dot{T}_{a}+\frac{1}{2}{ }^{(3)} \dot{T}_{a}\right) \\
& +\frac{1}{2} \dot{N}_{a b} \wedge^{(1)} \lambda^{a b}+\dot{R}_{a}{ }^{b} \wedge^{(2)} \lambda^{a}{ }_{b},
\end{aligned}
$$

where the three irreducible pieces of the torsion $\stackrel{(A)}{T}^{a}=$ (1) $\stackrel{(A)}{T} a+(2) \stackrel{(A)}{T} a+(3) \stackrel{(A)}{T} a$ under $S O(1,3)$-decomposition are

$$
\begin{aligned}
& \text { (1) } \stackrel{(A)}{T} a \text { : }=\stackrel{(A)}{T}^{a}-{ }^{(2)} \stackrel{(A)}{T}^{a}-{ }^{(3)} \stackrel{(A)}{T}^{a}, \quad \text { (tentor); } \\
& \text { (2) } \left.\stackrel{(A)}{T}^{a}:=\frac{1}{3} \stackrel{(A)}{\vartheta}^{a} \wedge\left(\stackrel{(A)}{e}_{b}\right\rfloor \stackrel{(A)}{T}^{b}\right), \quad \text { (trator); } \\
& { }^{(3)} T^{a}:=-\frac{1}{3}{ }^{*}\left(\stackrel{(A)}{\vartheta}^{a} \wedge *\left(\stackrel{(A)}{T} b \wedge \stackrel{(A)}{\vartheta^{\vartheta}} b\right)\right), \quad \text { (axiator). }
\end{aligned}
$$

The projective transformation

$$
\stackrel{(A)}{\Gamma} \underset{a}{b} \longrightarrow \stackrel{(A)}{\Gamma} \underset{a}{b}+\delta_{a}^{b} P
$$

with arbitrary 1-form field $P$, leaves the Hilbert-Einstein type Lagrangian invariant, which leads to the connection determined up to a 1-form. Projectively related connections have the same (unparametrized) geodesics. So, only projectively invariant matter Lagrangians would be allowed. This necessitates abandoning this constraint, at the very least replacing semi-Riemannian geometry by Weyl's. Namely, to remove this constraint from the gravitational Lagrangians
(82) and (86), following [120, 121], we may lift the Lagrange multiplier $\lambda^{a b}$ and add a dilaton type massless scalar field to

$$
V^{\prime}=V+\frac{1}{2} d \Phi \wedge \star d \Phi
$$

in the context of the Weyl 1-form $\stackrel{(A)}{N}$, which is of the type of a gauge potential for dilations anyways:

$$
\begin{gathered}
V_{\mathrm{GR}}^{\prime}=-\frac{1}{4 æ}\left(\breve{R}^{a b} \wedge \breve{\eta}_{a b}+\beta \stackrel{(A)}{N} \wedge \star \stackrel{(A)}{N}\right)+\stackrel{(A)}{T} a \wedge^{(2)} \lambda_{a}, \\
V_{\mathrm{EC}}^{\prime}=-\frac{1}{4 æ}\left(\stackrel{(A)}{R} a b \wedge \stackrel{(A)}{\eta^{\prime}} a b \stackrel{(A)}{N} \wedge \star \stackrel{(A)}{N}\right),
\end{gathered}
$$

respectively, provided the trace $\stackrel{(A)}{\Gamma}_{c} c$ of a connection is closely related to the Weyl 1-form $[17,18]$, as

$$
\begin{gathered}
\stackrel{(A)}{\Gamma}_{c}^{c}=2 \stackrel{(A)}{N}+d \ln \sqrt{\left|\operatorname{det} \stackrel{(A)}{g}_{a b}\right|}, \\
d \stackrel{(A)}{\Gamma}{ }_{c}^{c}=\stackrel{(A)}{R}_{c}^{c}=2 d \stackrel{(A)}{N} .
\end{gathered}
$$

In case if matter is present and supplies energy-momentum and hypermomentum currents, then the hypermomentum, via the second field equation (77), turns out to be proportional to the post-Riemannian pieces of the connection.

In case of translational gauge with the most general term $V=d{ }_{\vartheta}^{(A)} a \wedge \stackrel{(A)}{H}_{a}$, quadratic in $\stackrel{(A)}{\vartheta}^{a}$, the field equation $\delta L_{\text {tot }} / \delta_{\mathscr{\vartheta}}^{(A)} a=0$ then becomes

$$
\frac{\partial \stackrel{(A)}{\vartheta} a}{\partial \stackrel{(\pi)}{\vartheta} a^{\prime}} \wedge\left[d \stackrel{(A)}{H}_{a^{\prime}}-\stackrel{(A)}{E} a_{a^{\prime}}\right]=\stackrel{(A)}{\theta_{a}}
$$

where $\stackrel{(A)}{H}_{a}$ is linear in $\stackrel{(A)}{\vartheta}^{a}$, and

$$
\begin{aligned}
\stackrel{(A)}{E}_{a} & \left.\left.=\left(\stackrel{(A)}{e}_{a}\right\rfloor d \stackrel{(A)}{\vartheta} b\right) \wedge \stackrel{(A)}{H}_{b}-\frac{1}{2} \stackrel{(A)}{e}_{a}\right\rfloor(d \stackrel{(A)}{\mathscr{\vartheta}} b \wedge \stackrel{(A)}{H} b) \\
& \left.\left.=\frac{1}{2}\left[\left(\stackrel{(A)}{e}_{a}\right\rfloor d \stackrel{(A)}{\vartheta} b\right) \wedge \stackrel{(A)}{H}_{b}-d \stackrel{(A)}{\mathcal{\vartheta}} b \wedge\left(\stackrel{(A)}{e}_{a}\right\rfloor \stackrel{(A)}{H}_{b}\right)\right],
\end{aligned}
$$

the energy-momentum current of the gauge field. This can be considered as a starting point for turning to the Lagrangians with the quadratic ansatz for the kinetic term, that is, quadratic in the field strengths. Note that recently the authors of [122] show that the inverse of the so-called BarberoImmirzi parameter multiplying the pseudoscalar curvature, because of the topological Nieh-Yan form, can only be appropriately discussed if torsion square pieces are included. Using more conservative direct constructions, they establish the exact relations between both approaches by applying the topological Euler and Pontryagin forms in a Riemann-Cartan space expressed for the first time in terms of irreducible 
pieces of the curvature tensor. Only in a Riemann-Cartan spacetime, that is, in a spacetime with torsion, parity violating terms can be brought into the gravitational Lagrangian in a straightforward and natural way. They conclude that Riemann-Cartan spacetime is a natural habitat for chiral fermionic matter fields. However, the standard EC theory is invariant under the spacetime diffeomorphisms and local $S O(3,1)$ transformations acting in the tangent space. In our approach we suggest extending the $S O(3,1)$ to local $G L(4, R)$ group. This group is surely not the invariance of the action of standard theory, and so it takes the latter into diverse other ones, still invariant under spacetime diffeomorphisms.

\section{Concluding Remarks}

The actual advantage of present approach is twofold. To complete the TSSD theory [82], at first, we build up the distortion-complex and show how it restores the worlddeformation tensor, which still has been put in it by hand. In this approach we suggest to extend the $S O(3,1)$ group of standard EC theory to local $G L(4, R)$ group. The latter is surely not the invariance of the action of standard EC theory, and so it takes the latter into diverse other ones still invariant under spacetime diffeomorphisms. All the fundamental gravitational structures in fact, the metric as much as the coframes and connections, acquire a DC induced theoretical interpretation. Through a nontrivial choice of explicit form of a DC which we have at our disposal, we have a way to construct various generalizations and alternatives of the standard gravity. Secondly, we extend the geometrical ideas of the TSSD theory to study, in the framework of the first order Lagrangian, the physical foundation of the gauge model of more general metric-affine gravity theory with a dynamical torsion. It is remarkable that, in the framework of the first order standard Lagrangian, the equations of the standard MAG theory, in which the equation defining torsion is the algebraic type and in fact no propagation of torsion is allowed, can be equivalently replaced by the modified equations in which the torsion, in general, is dynamical. In testing the too general MAG equations (77) in some limit, we have to put on Lagrange multipliers to recover the field equation for different (sub)cases: in the case of TSSD-PG, one has to kill nonmetricity; TSSD-EC is the TSSD-PG with the curvature scalar as gravitational Lagrangian, whereas the special physical ansatz for the spacetime deformations yields the short-range propagating spin-spin interaction.

\section{Appendices}

\section{A. TSSD: More Details}

For a local spacetime deformation $\dot{\pi}(\dot{x})$, we have

$$
\begin{aligned}
& \dot{\Omega}_{b}{ }^{a}=\dot{\pi}_{c}{ }^{a} \dot{\pi}_{b}{ }^{c}=\dot{\Omega}_{\mu}{ }^{\nu} \breve{e}^{a}{ }_{\nu} \breve{e}_{b}{ }^{\mu}, \quad \dot{\Omega}_{\mu}{ }^{\nu}=\dot{\pi}_{\mu}{ }^{\rho} \dot{\pi}_{\rho}{ }^{\nu}, \\
& \dot{e}_{\rho}=\dot{\pi}_{\rho}{ }^{\nu} \breve{e}_{v} \equiv \dot{\partial}_{\rho}=\frac{\partial}{\partial \dot{x}^{\rho}}, \quad \dot{\vartheta}^{\rho}=\dot{\pi}^{\rho}{ }_{\mu} \breve{\vartheta}^{\mu} \equiv d \dot{x}^{\rho} .
\end{aligned}
$$

Since we are interested only in a peculiar condition (53) to be held, then it is completely satisfactory for further consideration to write the first deformation matrix, $\dot{\pi}(\dot{x})$, in the form of a particular solution to (53). To derive the particular solution to (53), we recall that, for an arbitrary matrix $M$,

$$
\operatorname{tr}\left\{M^{-1} \partial_{\mu} M\right\}=\partial_{\mu} \ln |M|
$$

where $|\cdots|$ denotes the determinant and tr the trace. According to it, in matrix notation $\breve{\omega}_{\mu}:=\left(\breve{\omega}^{a}{ }_{b \mu}\right),(53)$ becomes

$$
\operatorname{tr}\left\{\dot{\pi}(\dot{x}) \dot{\partial}_{\mu} \dot{\pi}^{-1}(\dot{x})\right\}=-\dot{\partial}_{\mu} \ln |\dot{\pi}(\dot{x})|=\operatorname{tr} \breve{\omega}_{\mu}(\breve{x}),
$$

which gives

$$
|\dot{\pi}(\dot{x})|=|\dot{\pi}(0)| \exp \left\{-\int_{0}^{\dot{x}} \operatorname{tr} \breve{\omega}_{\mu}(\breve{x}) d \dot{x}^{\prime \mu}\right\} .
$$

A particular solution to (53) is then (55). The first deformation matrices $\sigma(x):=\left(\sigma_{b}{ }^{a}\right)(x)$ contribute to corresponding ingredient part, $\chi_{b}{ }^{d}$, of the general deformation tensor,

$$
\begin{gathered}
\Omega_{b}{ }^{a}=\chi_{b}{ }^{d} \dot{\Omega}_{d}{ }^{a}=\chi_{b}{ }^{d} \dot{\Omega}_{\rho}{ }^{v} \breve{e}_{v}{ }^{a} \breve{e}^{\rho}{ }_{d}, \\
\bar{\chi}_{d}{ }^{c}=\sigma_{e}{ }^{c} \sigma_{d}{ }^{e}, \quad \bar{\chi}_{e}{ }^{d} \dot{\pi}_{b}{ }^{e}=\chi_{b}{ }^{e} \dot{\pi}_{e}{ }^{d},
\end{gathered}
$$

or

$$
\Omega_{\mu}{ }^{v}=\chi_{\mu}{ }^{\rho} \dot{\Omega}_{\rho}{ }^{v}, \quad \chi_{\mu}{ }^{\rho}=\chi_{b}{ }^{d} \breve{e}_{d}{ }^{\rho} \breve{e}_{\mu}^{b} \text {. }
$$

The tetrad changes according to

$$
\begin{gathered}
e_{c}=\left(\sigma_{c}^{d} \dot{\pi}_{d}^{a}\right) \breve{e}_{a}=\sigma_{c}{ }^{d} \dot{e}_{d}, \\
\vartheta^{c}=\left(\sigma^{c} \dot{\pi}^{e}{ }_{b}\right) \breve{\vartheta}^{b}=\sigma^{c}{ }_{e} \dot{\vartheta}^{e}, \quad e_{\rho}=\sigma_{\rho}{ }^{\sigma} \dot{e}_{\sigma}, \\
\vartheta^{\rho}=\sigma_{\sigma}{ }^{\rho} \dot{\vartheta}^{\sigma}, \quad e_{\rho}=\sigma_{\rho}{ }^{c} \dot{e}_{c}, \quad \vartheta^{\rho}=\sigma_{c}{ }^{\rho} \dot{\vartheta}^{c}, \\
\sigma_{\rho}^{c}=\sigma_{\rho}{ }^{\sigma} \dot{e}_{\sigma}^{c}, \quad \sigma_{c}^{\rho}{ }_{c}^{c}=\sigma_{\sigma}^{\rho} \dot{e}_{c}^{\sigma} .
\end{gathered}
$$

The corresponding second deformation matrices read

$$
\begin{gathered}
\gamma_{c d}(x)=\bar{\chi}_{e e^{\prime}} \dot{\pi}_{c}{ }^{e} \dot{\pi}_{d} e^{\prime}, \\
\dot{\gamma}_{c d}(\dot{x})=o_{a b} \dot{\pi}_{c}{ }^{a}(\dot{x}) \dot{\pi}_{d}{ }^{b}(\dot{x}),
\end{gathered}
$$

where $\bar{\chi}_{e e^{\prime}}=o_{a b} \sigma_{e}{ }^{a} \sigma_{e^{\prime}}^{b}$.

If the nonmetricity tensor $N_{\lambda \mu \nu}=-\mathscr{D}_{\lambda} g_{\mu \nu} \equiv-g_{\mu \nu ; \lambda}$ does not vanish, the general formula for the affine connection written in the spacetime components is [94]

$$
\stackrel{(A)}{\Gamma}_{\mu \nu}=\breve{\Gamma}_{\mu \nu}^{\rho}+\stackrel{(A)}{K}_{\mu}^{\rho}{ }_{\mu \nu}-\stackrel{(A)}{N}^{\rho}{ }_{\mu \nu}+\frac{1}{2} \stackrel{(A)}{N}_{(\mu \nu)}^{\rho} .
$$


Here $\stackrel{(A)}{K}_{\mu \nu}:=2 \stackrel{(A)}{Q}_{(\mu \nu)}^{\rho}+\stackrel{(A)}{Q}^{\rho}{ }_{\mu \nu}$ is the non-Riemann part-

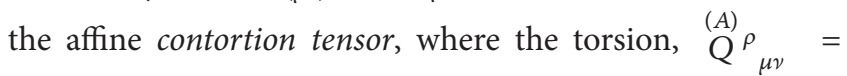
$(1 / 2) \stackrel{(A)}{T}_{\mu \nu}=\stackrel{(A)}{\Gamma}^{\rho}{ }_{[\mu \nu]}$ given with respect to a holonomic frame, $d \stackrel{(A)}{\vartheta} \rho=0$, is a third-rank tensor, antisymmetric in the first two indices, with 24 independent components. We may introduce the contortion tensors related to the deformation-related frame connection (60) and the spin connection (62):

$$
\stackrel{(A)}{K}_{a v}=\stackrel{(A)}{\omega}_{a v}+\stackrel{(A)}{\Delta}^{c} \text { av }
$$

where

$$
\stackrel{(A)}{\Delta}_{\mu \rho \nu}=\stackrel{(A)}{e}_{\mu a} \stackrel{(A)}{e}_{[\rho, \nu]}-\stackrel{(A)}{e}_{\rho a} \stackrel{(A)}{e}_{[\mu, \nu]}-\stackrel{(A)}{e}_{\nu a} \stackrel{(A)}{e}_{[\mu, \rho]}
$$

is referred to as the the Ricci coefficients of rotation. Both the contortion tensor and spin connection are antisymmetric in their first two indices. The Levi-Civita spin connection

$$
\breve{\omega}_{a \rho}^{\mu}=\stackrel{(\pi)}{e}_{a: \rho}^{\mu}=\stackrel{(\pi)}{\mu}_{a, \rho}^{\mu}+\breve{\Gamma}_{v \rho}^{\mu} \stackrel{(\pi)}{e}_{a}
$$

is related to the Ricci rotation coefficients, with $\stackrel{(A)}{K}=0$; thus,

$$
\stackrel{(A)}{K}_{a \rho}^{\mu}=\stackrel{(A)}{\omega}_{a \rho}^{\mu}-\breve{\omega}_{a \rho}^{\mu} .
$$

The relations between the corresponding torsion and contortion tensors read

$$
\stackrel{(A)}{K}_{\mu \nu}:=2 \stackrel{(A)}{Q}_{(\mu \nu)}^{\rho}+\stackrel{(A)}{Q}^{\rho}{ }_{\mu \nu}, \quad \stackrel{(A)}{Q}_{\mu \nu}{ }_{\mu \nu} \stackrel{(A)}{K}^{\rho}[\mu \nu]
$$

where

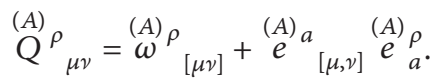

So, the affine connection (65) reads

$$
\Gamma_{\rho \sigma}^{\mu}=\stackrel{(\sigma)}{\Gamma}_{(\rho \sigma)}^{\mu}+\stackrel{(\sigma)}{Q}_{\rho \sigma}^{\mu}=\stackrel{(\pi)}{\Gamma}^{\mu}{ }_{(\rho \sigma)}+\stackrel{(\pi)}{Q}_{\rho \sigma}^{\mu}
$$

where $\stackrel{(A)}{\Gamma}^{\mu}{ }_{(\rho \sigma)}=\breve{\Gamma}_{(\rho \sigma)}^{\mu}+2{\stackrel{(A)^{\mu}}{(\rho \sigma)}}_{(\rho)}$. In the presence of curvature and torsion, the coupling prescription of a general field carrying an arbitrary representation of the Lorentz group will be

$$
\partial_{\mu} \longrightarrow \stackrel{(A)}{\mathscr{D}}_{\mu}=\partial_{\mu}-\frac{i}{2}\left(\stackrel{(A)}{\omega}_{\mu b}-\stackrel{(A)}{K}_{\mu b}^{a b}\right) J_{a b} .
$$

\section{B. Some Algebraic Operations}

Since the $4 \mathrm{D}$-dimensional teleparallel manifold $\mathscr{M}_{4}$ is diffeomorphic to $R^{4}$, one can choose an orientation on $\mathscr{M}_{4}$ and restrict the frames to agree with that orientation so that only transformations with values in $G L_{4}^{+}(R)$ are allowed. The metric then defines the Hodge dual of differential forms. The following algebraic operations are defined.
The Hodge dual map $\star: \Omega^{p} \rightarrow \Omega^{n-p}$. This is a $C^{\infty}$ linear map $\star: \Omega^{p} \rightarrow \Omega^{n-p}$, which acts on the wedge product monomials of the basis 1 -forms as

$$
\star\left(\vartheta^{a_{1} \cdots a_{p}}\right)=\varepsilon^{a_{1} \cdots a_{n}} e_{a_{p+1} \cdots a_{n}},
$$

where $e_{a_{i}}(i=p+1, \ldots, n)$ are understood as the downindexed 1-forms $e_{a_{i}}=o_{a_{i} b} \vartheta^{b}$ and $\epsilon^{a_{1} \cdots a_{n}}$ is the total antisymmetric pseudotensor. A further relation involving Hodge duality reads $\left.{ }^{\star}\left(\alpha \wedge e_{a}\right)=\left(e_{a}\right\rfloor^{\star} \alpha\right)$, while for differential forms $\alpha, \beta$ of the same degree $p$, equation ${ }^{\star}(\alpha \wedge \beta)={ }^{\star}(\beta \wedge \alpha)$ holds.

The eta basis is consisting in the Hodge dual of exterior products of tetrads by means of the Levi-Civita object: $\eta^{a b c d}:={ }^{\star} 9^{a b c d}$, which yields

$$
\begin{aligned}
\eta^{a b c} & :={ }^{\star} \vartheta^{a b c}=\eta_{d}^{a b c} \wedge \vartheta^{d}, \\
\eta^{a b} & :={ }^{\star} \vartheta^{a b}=\frac{1}{2 !} \eta_{c d}^{a b} \wedge \vartheta^{c d}, \\
\eta^{a} & :={ }^{\star} \vartheta^{a}=\frac{1}{3 !} \eta_{b c d}^{a} \wedge \vartheta^{b c d}, \\
\eta & :={ }^{\star} 1=\frac{1}{4 !} \eta_{a b c d} \vartheta^{a b c d},
\end{aligned}
$$

such that

$$
\begin{gathered}
\vartheta^{e} \wedge \eta_{a b c d}=-\delta_{a}^{e} \eta_{b c d}+\delta_{d}^{e} \eta_{a b c}-\delta_{c}^{e} \eta_{d a b}+\delta_{b}^{e} \eta_{c d a}, \\
\vartheta^{e} \wedge \eta_{a b c}=\delta_{a}^{e} \eta_{b c}+\delta_{c}^{e} o_{a b}+\delta_{b}^{e} \eta_{c a}, \\
\vartheta^{e} \wedge o_{a b}=-\delta_{a}^{e} \eta_{b}+\delta_{b}^{e} \eta_{a}, \quad \vartheta^{e} \wedge \eta_{a}=\delta_{a}^{e} \eta .
\end{gathered}
$$

\section{Conflict of Interests}

The author declares that there is no conflict of interests regarding the publication of this paper.

\section{Acknowledgments}

The author would like to thank W.-T. Ni for drawing attention to the pseudoscalar-photon interaction theory. This paper was only made possible through substantial support of the anonymous referees whose helpful and knowledgable comments and suggestions are much appreciated.

\section{References}

[1] R. Utiyama, "Invariant theoretical interpretation of interaction," Physical Review Letters, Physical Review, and Reviews of Modern Physics, vol. 101, pp. 1597-1607, 1956.

[2] T. W. B. Kibble, "Lorentz invariance and the gravitational field," Journal of Mathematical Physics, vol. 2, pp. 212-221, 1961.

[3] D. W. Sciama, "On the analog between charge and spin in general relativity," in Recent Developments in General Relativity, Festschrift for Leopold Infeld, p. 415, Pergamon Press, New York, NY, USA, 1962.

[4] D. W. Sciama, "The physical structure of general relativity," Reviews of Modern Physics, vol. 36, no. 1, pp. 463-469, 1964.

[5] A. Papapetrou, "Einstein's theory of gravitation and flat space," Proceedings of the Royal Irish Academy, vol. 52, pp. 11-23, 1948. 
[6] A. Papapetrou, "Spinning test-particles in general relativity. I," Proceedings of the Royal Society A, vol. 209, no. 1097, pp. 248258, 1951.

[7] A. Papapetrou, Lectures on General Relativity, Reidel D, 1974.

[8] A. Trautman, "On the structure of the EinsteinCartan equations," in Differential Geometry, vol. 12 of Symposia Mathematica, pp. 139-162, Academic Press, London, UK, 1973.

[9] J.-P. Francoise, G. L. Naber, and S. T. Tsou, Eds., Encyclopedia of Mathematical Physics, vol. 2, Elsevier, Oxford, UK, 2006.

[10] B. Kuchowicz, "Two-component neutrino field and spherical symmetry in a space-time with curvature and torsion," Physics Letters A, vol. 50, no. 4, pp. 267-268, 1974.

[11] K. S. Stelle and P. C. West, "De Sitter gauge invariance and the geometry of the Einstein-Cartan theory," Journal of Physics A: Mathematical and General, vol. 12, no. 8, pp. L205-L210, 1979.

[12] K. S. Stelle and P. C. West, "Spontaneously broken de Sitter symmetry and the gravitational holonomy group," Physical Review D, vol. 21, no. 6, pp. 1466-1488, 1980.

[13] T. Eguchi, P. B. Gilkey, and A. J. Hanson, "Gravitation, gauge theories and differential geometry," Physics Reports C, vol. 66, no. 6, pp. 213-393, 1980.

[14] F. W. Hehl and B. K. Datta, "Nonlinear spinor equation and asymmetric connection in general relativity," Journal of Mathematical Physics, vol. 12, pp. 1334-1339, 1971.

[15] F. W. Hehl, P. von der Heyde, G. D. Kerlick, and J. M. Nester, "General relativity with spin and torsion: foundations and prospect," Reviews of Modern Physics, vol. 48, no. 3, pp. 393-416, 1976.

[16] F. W. Hehl and J. D. McCrea, "Bianchi identities and the automatic conservation of energy-momentum and angular momentum in general-relativistic field theories," Foundations of Physics, vol. 16, no. 3, pp. 267-293, 1986.

[17] F. W. Hehl, E. A. Lord, and L. L. Smalley, "Metric-affine variational principles in general relativity II. Relaxation of the RIEmannian constraint," General Relativity and Gravitation, vol. 13, no. 11, pp. 1037-1056, 1981.

[18] F. W. Hehl, J. D. McCrea, E. W. Mielke, and Y. Ne'eman, "Metric-affine gauge theory of gravity: field equations, Noether identities, world spinors, and breaking of dilation invariance," Physics Reports, vol. 258, no. 1-2, pp. 1-171, 1995.

[19] A. B. Borisov and V. I. Ogievetskii, "Theory of dynamical affine and conformal symmetries as the theory of the gravitational field," Theoretical and Mathematical Physics, vol. 21, no. 3, pp. 1179-1188, 1974.

[20] S. W. MacDowell and F. Mansouri, "Unified geometric theory of gravity and supergravity," Physical Review Letters, vol. 38, no. 14, pp. 739-742, 1977.

[21] F. Mansouri and L. N. Chang, "Gravitation as a gauge theory," Physical Review D, vol. 13, no. 12, pp. 3192-3200, 1976.

[22] F. Mansouri, "Conformal gravity as a gauge theory," Physical Review Letters, vol. 42, no. 16, pp. 1021-1024, 1979.

[23] M. Schweizer and N. Straumann, "Poincaré gauge theory of gravitation and the binary pulsar $1913+16$," Physics Letters A, vol. 71, no. 5-6, pp. 493-495, 1979.

[24] M. Schweizer, N. Straumann, and A. Wipf, "Post-Newtonian generation of gravitational waves in a theory of gravity with torsion," General Relativity and Gravitation, vol. 12, no. 11, pp. 951-961, 1980.

[25] G. Grignani and G. Nardelli, "Gravity and the Poincaré group," Physical Review D, vol. 45, no. 8, pp. 2719-2731, 1992.
[26] L. N. Chang and F. Mansouri, "Nonlinear gauge fields and the structure of gravity and supergravity theories," Physical Review D, vol. 17, no. 12, pp. 3168-3178, 1978.

[27] E. A. Ivanov and V. I. Ogievetsky, "Gauge theories as theories of spontaneous breakdown," Letters in Mathematical Physics, vol. 1, no. 4, pp. 309-313, 1976.

[28] Y. Ne'eman and T. Regge, "Gravity and supergravity as gauge theories on a group manifold," Physics Letters B, vol. 74, no. 1-2, pp. 54-56, 1978.

[29] Y. Neeman and D. J. Sijacki, "Unified affine gauge theory of gravity and strong interactions with finite and infinite overline GL(4, R) spinor fields," Annals of Physics, vol. 120, pp. 292-315, 1979.

[30] Y. Ne’eman and D. Šijački, "World spinors-construction and some applications," Foundations of Physics, vol. 27, no. 8, pp. 1105-1122, 1997.

[31] M. Toller, "Classical field theory in the space of reference frames," II Nuovo Cimento B, vol. 44, no. 1, pp. 67-98, 1978.

[32] G. Cognola, E. Soldati, M. Toller, L. Vanzo, and S. Zerbini, "Theories of gravitation in the space of reference frames," Il Nuovo Cimento B, vol. 54, no. 2, pp. 325-348, 1979.

[33] J. Niederle and E. A. Ivanov, "Gauge formulation of gravitation theories. I. The Poincaré, de Sitter, and conformal cases," Physical Review D: Particles and Fields, vol. 25, no. 4, pp. 976987, 1982.

[34] P. G. Bergmann, V. de Sabbata, G. T. Gillies, P. I. Pronin, and A. Zichichi, Eds., Spin in Gravity: Is It Possible to Give an Experimental Basis to Torsion?: International School of Cosmology and Gravitation XV Course Erice, Italy 13-20 May 1997, World Scientific, Singapore, 1997.

[35] V. de Sabbata and M. Gasperini, "Introduction to gravitation," in Unified Field Theories of More Than Four Dimensions, V. de Sabbata and E. Schmutzer, Eds., World Scientific Publishers, 1985.

[36] V. de Sabbata and M. Gasperini, "On the Maxwell equations in a Riemann-Cartan space," Physics Letters A, vol. 77, no. 5, pp. 300-302, 1980.

[37] V. de Sabbata and C. Sivaram, Spin and Torsion in Gravitation, World Scientific Publishers, Singapore, 1994.

[38] J. Nitsch and F. W. Hehl, "Translational gauge theory of gravity: post-newtonian approximation and spin precession," Physics Letters B, vol. 90, no. 1-2, pp. 98-102, 1980.

[39] D. Ivanenko and G. A. Sardanashvily, "The gauge treatment of gravity," Physics Reports, vol. 94, no. 1, pp. 1-45, 1983.

[40] T. W. B. Kibble and K. S. Stelle, "Gauge theories of gravity and supergravity," in Progress in Quantum Field Theory, $\mathrm{H}$. Ezava and S. Kamefuchi, Eds., p. 57, Elsevier, Amsterdam, The Netherlands, 1986.

[41] C. Wiesendanger, "Poincaré gauge invariance and gravitation in Minkowski spacetime," vol. 13, no. 4, pp. 681-700, 1996.

[42] F. Gronwald and F. W. Hehl, "On the gauge aspects of gravity," http://arxiv.org/abs/gr-qc/9602013.

[43] E. Cartan, "On a generalization of the notion of Riemann curvature and spaces with torsion," Comptes Rendus de l'Académie des Sciences, vol. 174, pp. 593-595, 1922.

[44] E. Cartan, "On manifolds with an affine connection and the theory of general relativity," Annales Scientifiques de l'École Normale Supérieure, vol. 40, pp. 325-412, 1923.

[45] E. Cartan, "Sur les variétés à connexion affine et la théorie de la relativité généralisée (première partie suite)," Annales 
Scientifiques de l'École Normale Supérieure, vol. 41, pp. 1-25, 1924.

[46] E. Cartan, Sur les Variables a Connexion Affine et la Theorie de la Relativite Generalisee, Gauthier-Villard, Paris, France, 1955.

[47] Y. Ne'eman and F. W. Hehl, "Test matter in a spacetime with nonmetricity," Classical and Quantum Gravity, vol. 14, no. 1A, pp. A251-A259, 1997.

[48] E. W. Mielke, F. Gronwald, Y. N. Obukhov, R. Tresguerres, and F. W. Hehl, "Towards complete integrability of two-dimensional Poincaré gauge gravity," Physical Review D, vol. 48, no. 8, pp. 3648-3662, 1993.

[49] E. W. Mielke, Y. N. Obukhov, and F. W. Hehl, "Yang-Mills configurations from 3D Riemann-Cartan geometry," Physics Letters A, vol. 192, no. 2-4, pp. 153-162, 1994.

[50] E. W. Mielke, "Consistent coupling to Dirac fields in teleparallelism: comment on 'metric-afflne approach to teleparallel gravity,' Physical Review D, vol. 69, Article ID 128501, 2004.

[51] S. Sternberg, Semi-Riemannian Geometry and General Relativity, 2003, http://www.math.harvard.edu/ shlomo/docs/ semi_riemannian_geometry.pdf.

[52] M. Blagojevic, Gravitation and Gauge Symmetries, High Energy Physics, Cosmology and Gravitation, Edited by: B. Foster, L. Grishchuk , E. W. Kolb, M. A. H. MacCallum , D. H. Perkins and B. F. Schutz, 2002.

[53] E. A. Lord, "Poincaré gauge theory from self-coupling," Pramana, vol. 29, no. 4, pp. 359-368, 1987.

[54] R. T. Hammond, "Torsion gravity," Reports on Progress in Physics, vol. 65, no. 5, pp. 599-649, 2002.

[55] R. Tresguerres, "Unified description of interactions in terms of composite fiber bundles," Physical Review D, vol. 66, no. 6, Article ID 064025, 15 pages, 2002.

[56] R. Tresguerres, "Translations and dynamics," International Journal of Geometric Methods in Modern Physics, vol. 5, no. 6, pp. 905-946, 2008.

[57] A. Tiemblo and R. Tresguerres, "Gauge theories of gravity: the nonlinear framework," Recent Research Developments in Physics, vol. 5, p. 1255, 2004.

[58] S. Capozziello and M. de Laurentis, "Gravity from local Poincaré gauge invariance," International Journal of Geometric Methods in Modern Physics, vol. 6, no. 1, pp. 1-24, 2009.

[59] G. Basini and S. Capozziello, "A general covariant symplectic structure for gravitational, electromagnetic and Dirac fields," International Journal of Modern Physics D: Gravitation, Astrophysics, Cosmology, vol. 15, no. 4, pp. 583-602, 2006.

[60] S. A. Ali, C. Cafaro, S. Capozziello, and C. Corda, "On the Poincaré gauge theory of gravitation," International Journal of Theoretical Physics, vol. 48, no. 12, pp. 3426-3448, 2009.

[61] D. K. Wise, "MacDowell- mansouri gravity and cartan geometry," Classical and Quantum Gravity, vol. 27, no. 15, Article ID 155010, 2010.

[62] B. Coll, in Proceedings of the Spanish Relativity Meeting (ERES '98), Salamanca, Spain, 1998.

[63] B. Coll, J. Llosa, and D. Soler, "Three-dimensional metrics as deformations of a constant curvature metric," General Relativity and Gravitation, vol. 34, no. 2, pp. 269-282, 2002.

[64] J. Llosa and D. Soler, "Reference frames and rigid motions in relativity," Classical and Quantum Gravity, vol. 21, no. 13, pp. 3067-3094, 2004.

[65] J. Llosa and D. Soler, "On the degrees of freedom of a semiRiemannian metric," Classical and Quantum Gravity, vol. 22, no. 5, pp. 893-908, 2005.
[66] J. Llosa and J. Carot, "Flat deformation theorem and symmetries in spacetime," Classical and Quantum Gravity, vol. 26, no. 5, Article ID 055013, 2009.

[67] S. Capozziello and C. Stornaiolo, "Space-time deformations as extended conformal transformations," International Journal of Geometric Methods in Modern Physics, vol. 5, no. 2, pp. 185-195, 2008.

[68] J. Alfaro, D. Espriu, and D. Puigdomènech, "Spontaneous generation of geometry in four dimensions," Physical Review D, vol. 86, Article ID 025015, 2012.

[69] M. Blagojevic and F. W. Hehl, Eds., Gauge Theories of Gravitation: A Reader with Commentaries, Imperial College Press, 2013.

[70] S. Kobayashi and K. Nomizu, Foundations of Differential Geometry, Interscience, New York, NY, USA, 1963.

[71] J. Plebanski, "Forms and riemannian geometry," in Proceedings of the International School of Cosmology and Gravitation, Erice, Italy, May 1972.

[72] A. Trautman, Differential Geometry for Physicists, Stony Brook Lectures, Bibliopolis, Napoli, Italia, 1984.

[73] F. W. Hehl, Y. Ne'eman, J. Nitsch, and P. von der Heyde, "Shortrange confining component in a quadratic poincaré gauge theory of gravitation," Physics Letters B, vol. 78, no. 1, pp. 102106, 1978.

[74] D. E. Neville, "Gravity Lagrangian with ghost-free curvaturesquared terms," Physical Review D, vol. 18, no. 10, pp. 3535-3543, 1978.

[75] D. Neville, "Gravity theories with propagating torsion," Physical Review D, vol. 21, no. 4, pp. 867-873, 1980.

[76] D. E. Neville, "Spin-2 propagating torsion," Physical Review D, vol. 23, no. 6, pp. 1244-1249, 1981.

[77] E. Sezgin and P. van Nieuwenhuizen, "New ghost-free gravity Lagrangians with propagating torsion," Physical Review D: Particles and Fields, vol. 21, no. 12, pp. 3269-3280, 1980.

[78] D. D. Ivanenko, P. I. Pronin, and G. A. Sardanasvily, The Gauge Gravitational Theory, Moscow Publishing House, 1985, (Russian).

[79] V. N. Ponomariev, A. O. Barvinsky, and Y. N. Obukhov, Geometrodynamica l Methods and Gauge Approach to Gravitational Theory, Moscow, Russia, Atomizdat, 1985, (Russian).

[80] J. Daum and M. Reuter, "Einstein-Cartan gravity, asymptotic safety, and the running Immirzi parameter," Annals of Physics, vol. 334, pp. 351-419, 2013.

[81] E. W. Mielke, "Is Einstein-Cartan theory coupled to light Fermions asymptotically safe?” Journal of Gravity, vol. 2013, Article ID 812962, 5 pages, 2013.

[82] G. Ter-Kazarian, "Two-step spacetime deformation-induced dynamical torsion," Classical and Quantum Gravity, vol. 28, no. 5, Article ID 055003, 2011.

[83] R. Aldrovandi and J. G. Pereira, An Introduction to Geometrical Physics, World Scientific Publishers, Singapore, 1995.

[84] R. Aldrovandi and J. G. Pereira, An Introduction to Teleparallel Gravity, Instituto de Fisica Teorica, UNSEP, Sao Paulo, Brazil, 2007.

[85] K. Hayashi and T. Shirafuji, "New general relativity," Physical Review D: Particles and Fields, vol. 19, no. 12, pp. 3524-3553, 1979.

[86] H. I. Arcos, V. C. de Andrade, and J. G. Pereira, "Torsion and gravitation: a new view," International Journal of Modern Physics $D$, vol. 13, no. 5, pp. 807-818, 2004.

[87] H. I. Arcos and J. G. Pereira, “Torsion gravity”, International Journal of Modern Physics D, vol. 13, no. 10, pp. 2193-2240, 2004. 
[88] R. Aldrovandi, P. B. Barros, and J. G. Pereira, "Gravitation as anholonomy," General Relativity and Gravitation, vol. 35, no. 6, pp. 991-1005, 2003.

[89] R. Aldrovandi, J. G. Pereira, and K. H. Vu, "Gravitation: global formulation and quantum effects," Classical and Quantum Gravity, vol. 21, no. 1, pp. 51-62, 2004.

[90] R. Aldrovandi, J. G. Pereira, and K. H. Vu, "Gravitation without the equivalence principle," General Relativity and Gravitation, vol. 36, no. 1, pp. 101-110, 2004.

[91] R. Aldrovandi, L. C. T. Guillen, J. G. Pereira, and K. H. Vu, "Bringing together gravity and the quanta," in Proceedings of the Albert Einstein Century International Conference, pp. 18-22, Paris, France, July 2005.

[92] V. C. de Andrade and J. G. Pereira, "Gravitational Lorentz force and the description of the gravitational interaction," Physical Review D, vol. 56, no. 8, pp. 4689-4695, 1997.

[93] V. C. de Andrade, L. C. T. Guillen, and J. G. Pereira, "Teleparallel spin connection," Physical Review D, vol. 64, no. 2, Article ID 027502, 2001.

[94] N. J. Poplawski, "Propagating torsion in the Einstein frame," Journal of Mathematical Physics, vol. 47, no. 11, Article ID 112504, 7 pages, 2006.

[95] W.-T. Ni, "Searches for the role of spin and polarization in gravity," Reports on Progress in Physics, vol. 73, Article ID 056901, 2010

[96] W.-T. Ni, "A new theory of gravity," Physical Review D, vol. 7, no. 10, pp. 2880-2883, 1973.

[97] D. L. Lee, A. P. Lightman, and W. T. Ni, "Conservation laws and variational principles in metric theories of gravity," Physical Review D: Particles and Fields, vol. 10, pp. 1685-1700, 1974.

[98] W. T. Ni, "Equivalence principles and electromagnetism," Physical Review Letters, vol. 38, no. 7, pp. 301-304, 1977.

[99] W.-T. Ni, "Their empirical foundations, and the role of precision experiments to test them," in Proceedings of the International School and Symposium on Precision Measurement and Gravity Experiment, W.-T. Ni, Ed., p. 491, National Tsing Hua University, Taipei, Taiwan, 1983.

[100] A. B. Balakin and W.-T. Ni, "Non-minimal coupling of photons and axions," Classical and Quantum Gravity, vol. 27, no. 5, Article ID 055003, 2010.

[101] G. T. Ter-Kazarian, "Gravitation and inertia; a rearrangement of vacuum in gravity," Astrophysics and Space Science, vol. 327, no. 1, pp. 91-109, 2010.

[102] L. S. Pontryagin, Continous Groups, Nauka, Moscow, Russia, 1984.

[103] B. A. Dubrovin, S. P. Novikov, and A. T. Fomenko, Contemporary Geometry, Nauka, Moscow, Russia, 1986.

[104] S. Coleman, J. Wess, and B. Zumino, "Structure of phenomenological lagrangians. I," Physical Review, vol. 177, no. 5, pp. 22392247, 1969.

[105] C. G. Callan, S. Coleman, J. Wess, and B. Zumino, "Structure of phenomenological lagrangians. II," Physical Review, vol. 177, no. 5, pp. 2247-2250, 1969.

[106] S. Weinberg, Brandeis Lectures, The MIT Press, Cambridge, Mass, USA, 1970.

[107] A. Salam and J. Strathdee, "Nonlinear realizations. II. Conformal symmetry," Physical Review Letters, vol. 184, pp. 1760-1768, 1969.

[108] C. J. Isham, A. Salam, and J. Strathdee, "Nonlinear realizations of space-time symmetries. Scalar and tensor gravity," Annals of Physics, vol. 62, pp. 98-119, 1971.
[109] D. V. Volkov, "Phenomenological Lagrangians," Soviet Journal of Particles and Nuclei, vol. 4, pp. 1-17, 1973.

[110] V. I. Ogievetsky, "Nonlinear realizations of internal and spacetime symmetries," in Proceedings of the 10th Winter School of Theoretical Physics, Wroclaw, Poland, 1974.

[111] F. W. Hehl, J. Lemke, and E. W. Mielke, "Two lectures on fermions and gravity," in Geometry and Theoretical Physics, J. Debrus and A. C. Hirshfeld, Eds., pp. 56-140, Springer, Heidelberg, Germany, 1991.

[112] Y. N. Obukhov and J. G. Pereira, "Metric-affine approach to teleparallel gravity," Physical Review D, vol. 67, no. 4, 044016, 17 pages, 2003.

[113] Y. N. Obukhov and J. G. Pereira, "Lessons of spin and torsion: reply to "Consistent coupling to Dirac fields in teleparallelism", Physical Review D, vol. 69, Article ID 128502, 2004.

[114] J. W. Maluf, "Dirac spinor fields in the teleparallel gravity: comment on Metric-affine approach to teleparallel gravity," Physical Review D, vol. 67, Article ID 108501, 2003.

[115] I. M. Singer, "Some remarks on the Gribov ambiguity," Communications in Mathematical Physics, vol. 60, no. 1, pp. 7-12, 1978.

[116] R. Tresguerres, "Exact vacuum solutions of 4-dimensional metric-affine gauge theories of gravitation," Zeitschrift für Physik C Particles and Fields, vol. 65, no. 2, pp. 347-354, 1995.

[117] R. Tresguerres, "Exact static vacuum solution of fourdimensional metric-affine gravity with nontrivial torsion," Physics Letters A, vol. 200, no. 6, pp. 405-410, 1995.

[118] J. K. Ho, D. Chern, and J. M. Nester, "Some spherically symmetric exact solutions of the metric-affine gravity theory," Chinese Journal of Physics, vol. 35, pp. 640-650, 1997.

[119] W.-T. Ni, "Constraints on pseudoscalar-photon interaction from CMB polarization observation," Progress of Theoretical and Experimental Physics, vol. 172, no. 49, pp. 175-178, 2008.

[120] F. W. Hehl, G. D. Kerlick, and P. von der Heyde, "On a new metric affine theory of gravitation," Physics Letters B, vol. 63, no. 4, pp. 446-448, 1976.

[121] J. D. McCrea, F. W. Hehl, and E. W. Mielke, "Mapping Noether identities into Bianchi identities in general relativistic theories of gravity and in the field theory of static lattice defects," International Journal of Theoretical Physics, vol. 29, no. 11, pp. 1185-1206, 1990.

[122] P. Baekler and F. W. Hehl, "Beyond Einstein-Cartan gravity: quadratic torsion and curvature invariants with even and odd parity including all boundary terms," Classical and Quantum Gravity, vol. 28, no. 21, Article ID 215017, 2011. 

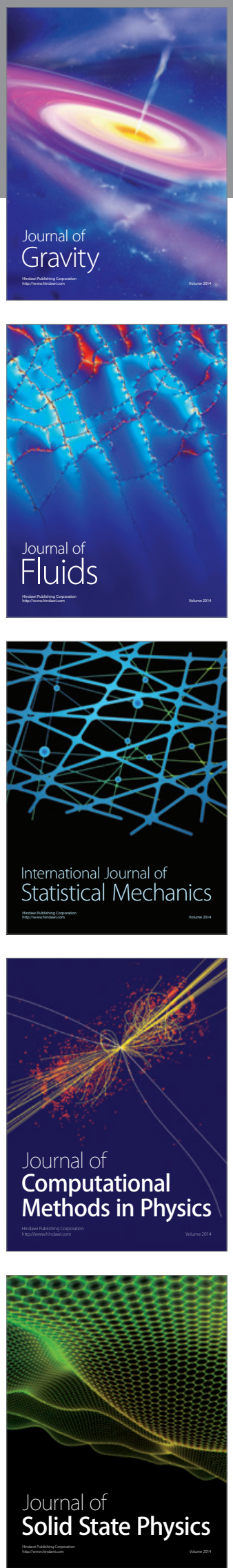

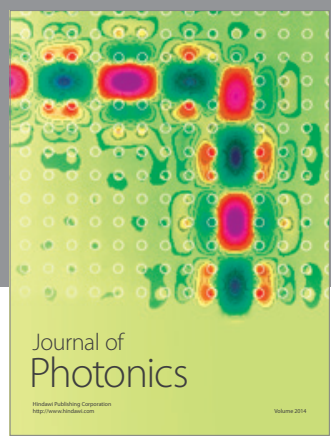

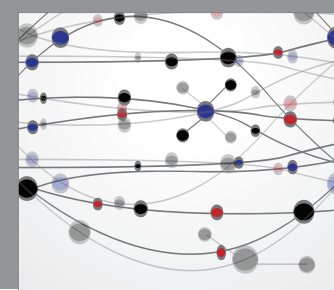

The Scientific World Journal

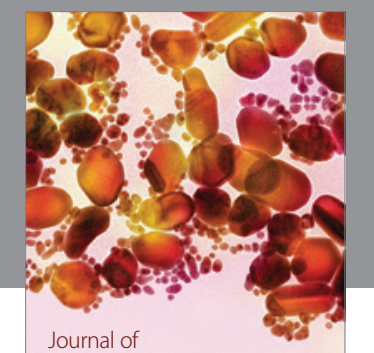

Soft Matter
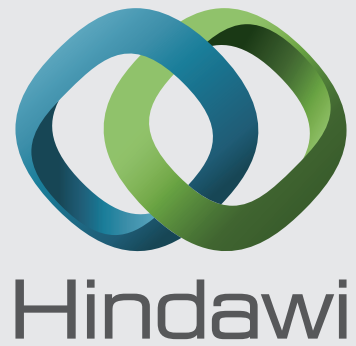

Submit your manuscripts at

http://www.hindawi.com
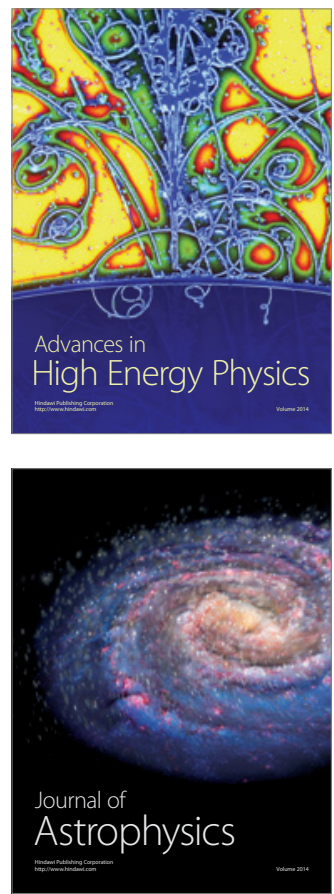
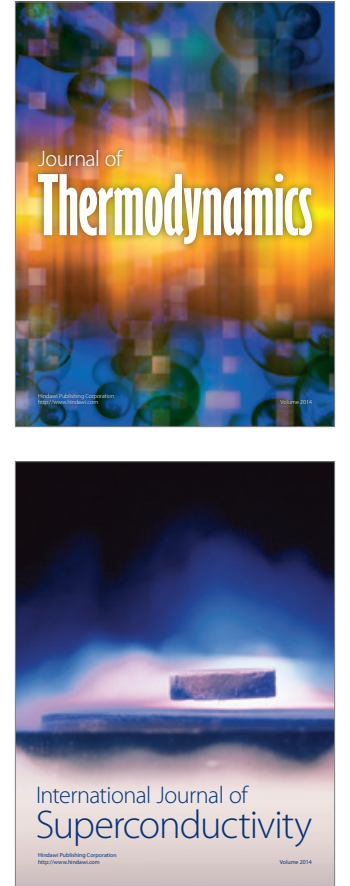
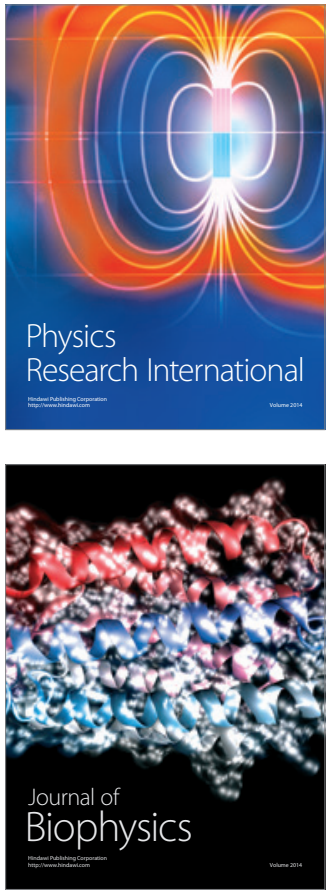
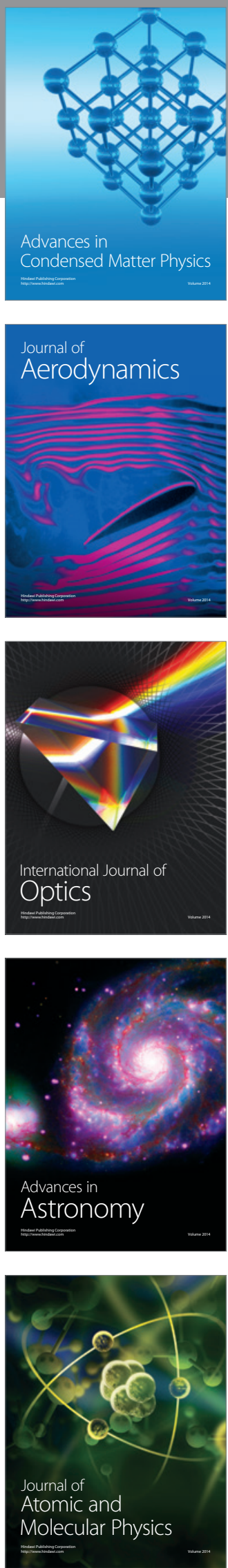